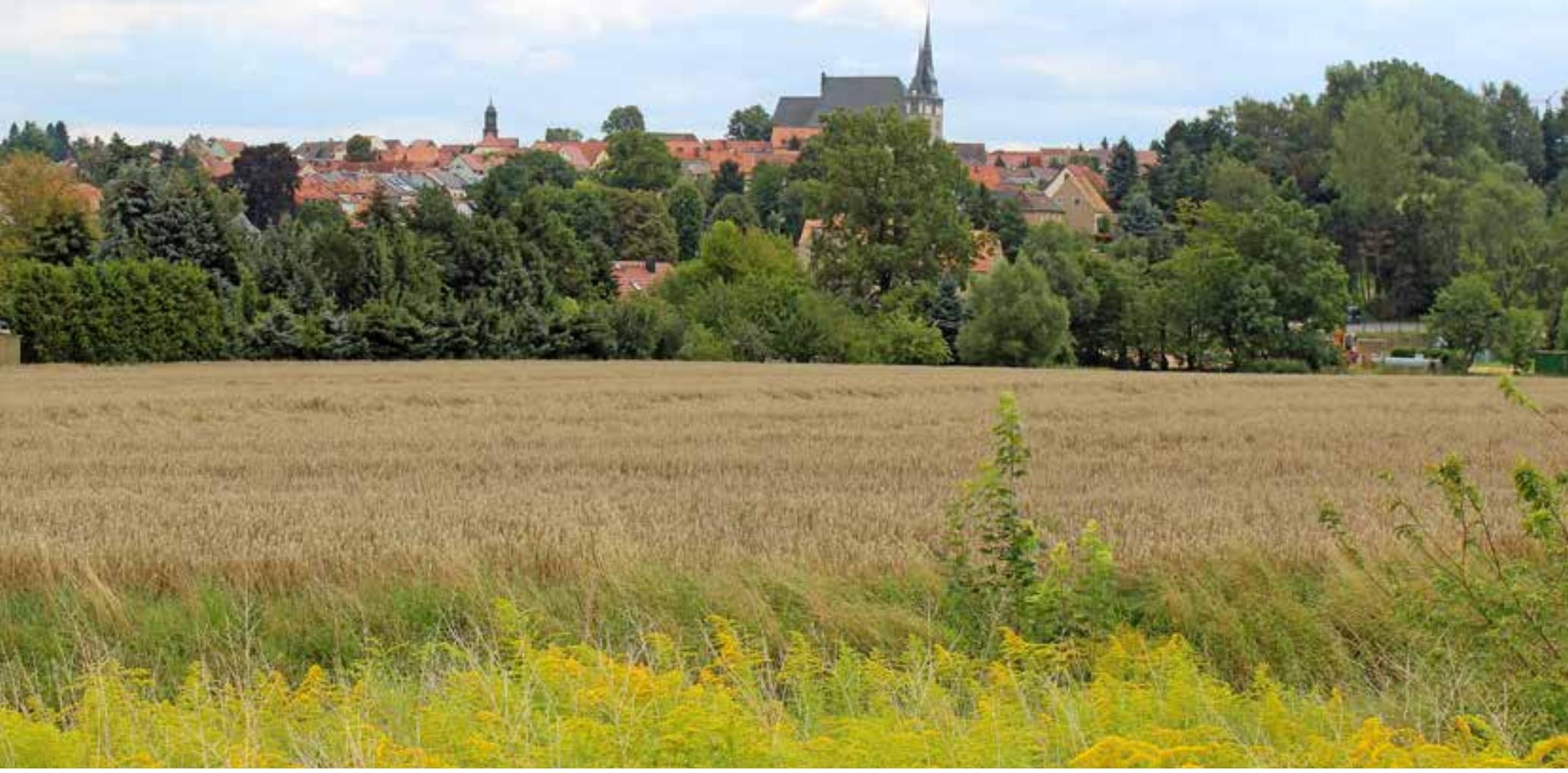

\title{
Lommatzsch
}

\section{Eine Skizze der Stadtentwicklung}

\section{Anita Maaß}

Eine knappe Überblicksdarstellung wie die folgende kann sich nur auf wenige Aspekte der über 700-jährigen Stadtgeschichte von Lommatzsch beschränken. Ziel ist es, die wichtigsten Entwicklungslinien der Kleinstadt im Zentrum der Lommatzscher Pflege zu skizzieren. Die chronologisch aufgebaute Beschreibung versucht, diese in ihre Rahmenbedingungen und Zusammenhänge einzuordnen. Allerdings gibt es - abgesehen von Ergebnissen archäologischer Grabungen ${ }^{1}$ - zur Stadtgeschichte von Lommatzsch keine aktuellen Forschungen. Dr. Hainer Plaul recherchiert zwar für ein „Stadt- und Landlexikon Lommatzsch und die Lommatzscher Pflege“, legte aber noch keine Ergebnisse vor. Anlässlich der 500-jährigen urkundlichen Ersterwähnung der Lommatzscher Pflege im Jahr 2017 beschäftigen sich aktuell zahlreiche Geschichtsinteressierte mit der His- torie der Lommatzscher Pflege. Neuere Ergebnisse zur Stadtgeschichte gibt es darunter bisher nicht. ${ }^{2}$ Die einzige Monografie zur Stadtgeschichte in den vergangen Jahren war ein Buch mit historischen Ansichten. ${ }^{3}$ Aus diesem Grund - und weil der Autorin ${ }^{4}$ aufgrund ihrer beruflichen Tätigkeit Zeit und Rahmenbedingungen für eigene Forschungen fehlen greift der vorliegende Beitrag auf die Sekundärliteratur zurück. Fundierte Quellenbezüge weisen Studien vom Ende des 19. und Anfang des 20. Jahrhunderts auf. ${ }^{5} 1979$ erschien eine heimatkundliche Bestandsaufnahme. ${ }^{6} 18$ Jahre später beschrieb Karlheinz Blaschke die Stadtentstehung von Lommatzsch und typisierte Lommatzsch als „Kirchenstadt“.7 Die aktuellen archäologischen Untersuchungen, die begleitend zu den innerstädtischen Baumaßnahmen erfolgen, unterziehen seine Theorie zwar nicht
Blick auf Lommatzsch Foto: Matthias Donath

1 Vgl. Oliver Spitzner/Michael Strobel: Zwei Jahre baubegleitende Untersuchungen in der Lommatzscher Innenstadt - eine Zwischenbilanz, in: Ausgrabungen in Sachsen, Heft $3 / 2012$, S. 183 187; vgl. auch zur Altsiedellandschaft Lommatzscher Pflege Michael Strobel/Richard Vogt/Thomas Westphalen: Die Lommatzscher Pflege - eine sächsische Altsiedellandschaft, in: Mitteilungen des Landesvereins Sächsischer Heimatschutz, Heft 2/2009, S. 4-12. 
2 Das Interesse an der Lommatzscher Stadtgeschichte besteht schon lange. In den 1930er Jahren veröffentlichte die „Lommatzscher Pflege. Heimatbeilage zum Lommatzscher Anzeiger und Tageblatt" in loser Folge Beiträge zur Heimatgeschichte. Allerdings standen diese unter dem Einfluss der nationalsozialistischen $\mathrm{Ge}$ schichtsschreibung und sind entsprechend quellenkritisch einzuordnen. Anlässlich der Stadt- und Schulfeste 1936, 1959 und 1986 entstanden Festschriften, die jedoch ebenfalls quellenkritisch mit Blick auf ihre Entstehungszeit einzuordnen sind. Anfang des 21. Jahrhunderts beschäftigten sich Lommatzscher Kinder- und Jugendliche mit ihrer Stadt, u. a. mit den Ereignissen im Frühjahr 1945 in Lommatzsch und Dörschnitz sowie mit der Geschichte der St. Wenzelskirche. Die Manuskripte aus den Jahren 2008 bis 2015, die die Konfirmanden im Projekt „Zeitensprünge“ unter Leitung des Pfarrers Roland Hartzsch (bis 2014 Pfarrer in Lommatzsch) erstellten, sind in der Kanzlei der evangelischen Kirchgemeinde Lommatzsch einsehbar.

3 Vgl. Gerth Skibinski: Lommatzsch. Ein historischer Stadtrundgang, Meißen 2011.

4 Die promovierte Historikerin Dr. Anita Maaß ist seit 2005 hauptamtliche Bürgermeisterin der Stadt Lommatzsch.

5 Vgl. Louis Zahn: Geschichtliches der Stadt Lommatzsch nebst einem Verzeichniß der daßigen Pastoren, Diakonen, Rektoren und Bürgermeister, Lommatzsch 1895; P. Schmidt: Die Parochie Lommatzsch, in: Neue Sächsische Kirchengalerie. Ephorie Meißen, Leipzig 1902, Sp. 9881044; vgl. insbesondere $\mathrm{zu}$ Bau- und Kunstdenkmalen: Cornelius Gurlitt: Beschreibende Darstellung der älteren Bau- und Kunstdenkmale in Sachsen. Bd. 41. Amtshauptmannschaft Meißen-Land, Dresden 1923 (Nachdruck Dresden 2014); S. 268-305. Nach Aussagen des Bürger-

Areal des früheren Paltzschener Sees bei Lommatzsch ๑ Wikimedia (Skjölding) der ausdrücklichen Prüfung, versuchen jedoch die bauliche Stadtentwicklung zu rekonstruieren. Dabei bestätigen die Funde die Bedeutung von Lommatzsch als überregionalem Wallfahrtsort in vorreformatorischer Zeit. ${ }^{8}$ Der vorliegende Aufsatz folgt damit der These von Blaschke, wonach in Lommatzsch das Vorhandensein einer Kirche „die Voraussetzung für die Entstehung der Stadt“ gewesen sei. ${ }^{9}$ Zugleich geht die Autorin der Frage nach, welche Faktoren die Entwicklung der Stadt Lommatzsch jeweils in ihrer historischen Epoche beförderten oder hemmten. Die Betrachtung gliedert sich in die Zeitabschnitte a) Mittelalter (10. bis 15. Jahrhundert), b) Neuzeit (16. bis 18. Jahrhundert), c) Industrialisierung (19./20. Jahrhundert) und d) Gegenwart (ab 1990), wobei aufgrund der Quellenlage und des Forschungsstandes keine Ausgewogenheit der historischen Darstellung möglich ist. Räumlich bezieht sich die Darstellung ausschließlich auf die Stadt Lommatzsch im engen Sinn ihrer Flurgrenzen. Die zugehörigen Dörfer ${ }^{10}$, die heute gemeinsam mit der Stadt die Gesamtkommune bilden, werden in ihrer Entwicklung nur dann einbezogen, wenn ein konkreter Zusammenhang mit der Stadtentwicklung bestand.

\section{Mittelalter (10. bis 15. Jahrhundert)}

Die Stadt Lommatzsch entwickelte sich im heutigen Gebiet der Lommatzscher Pflege. Der Landstrich gehört zum Mittelsächsischen Lößgebiet. Charakteristisch ist das sanft gewellte Hügelland, welches wasserführende Täler zerschneiden. Die Lößdecke des Bodens ist stark und nährstoffreich. Die steinarmen Lehmböden ließen sich bereits in der Jungsteinzeit mit ein- fachsten landwirtschaftlichen Geräten gut bearbeiten. ${ }^{11}$ Landschafts- und Ortsnamen erinnern noch heute an die Landnahme im frühen Mittelalter durch die Sorben. Am dichtesten besiedelt war der Gau Daleminze (Region heute westlich von Meißen bis Döbeln und Mügeln reichend) mit dem heutigen Lommatzsch in der Mitte und der Stammesburg Gana (vermutet bei Hof/ Stauchitz). ${ }^{12}$ Der heute nicht mehr vorhandene Paltzschener See bei Lommatzsch galt als Mittelpunkt und Heiligtum des religiösen Lebens der Daleminzier. Der Stadtname Lommatzsch sowie die vielen Dorfbezeichnungen in der Lommatzscher Pflege mit dem Suffix ,-itz“ verweisen auf diese slawischen Wurzeln. König Heinrich I. eroberte im Jahr 929 das Sorbenland und unterwarf die Daleminzier der deutschen Herrschaft. Mit der Gründung des Bistums Meißen 968 durch Kaiser Otto I. begann der Aufbau einer Kirchenorganisation. Mehrere kleine slawische Dörfer gehörten zu einer Pfarrkirche, von denen etliche im Gebiet gebaut wurden, darunter in der späteren Stadt Lommatzsch. ${ }^{13}$ Der Kirchenbau erfolgte damals eher ungewöhnlich auf einem 175 Meter hohen Hügel, der höchsten Stelle der Offenlandschaft. Damit befand sie sich jedoch in Sichtweite zum slawischen Heiligtum Paltzschener See, auch „GlommatzBrunnen“ genannt. ${ }^{14}$ Der See existierte bereits im 19. Jahrhundert nicht mehr. In der ältesten Überlieferung des Chronisten Thietmar von Merseburg heißt es: „Glomuzi ist eine Quelle, nicht über zwei Meilen weit von der Elbe entfernt; diese bildet einen stehenden See, der wie die Eingeborenen behaupten und viele Augenzeugen bestätigen, häufig wunderbare Erscheinungen zeigt. So lange holder Friede die Bewohner des Landes beglückt und der Boden die

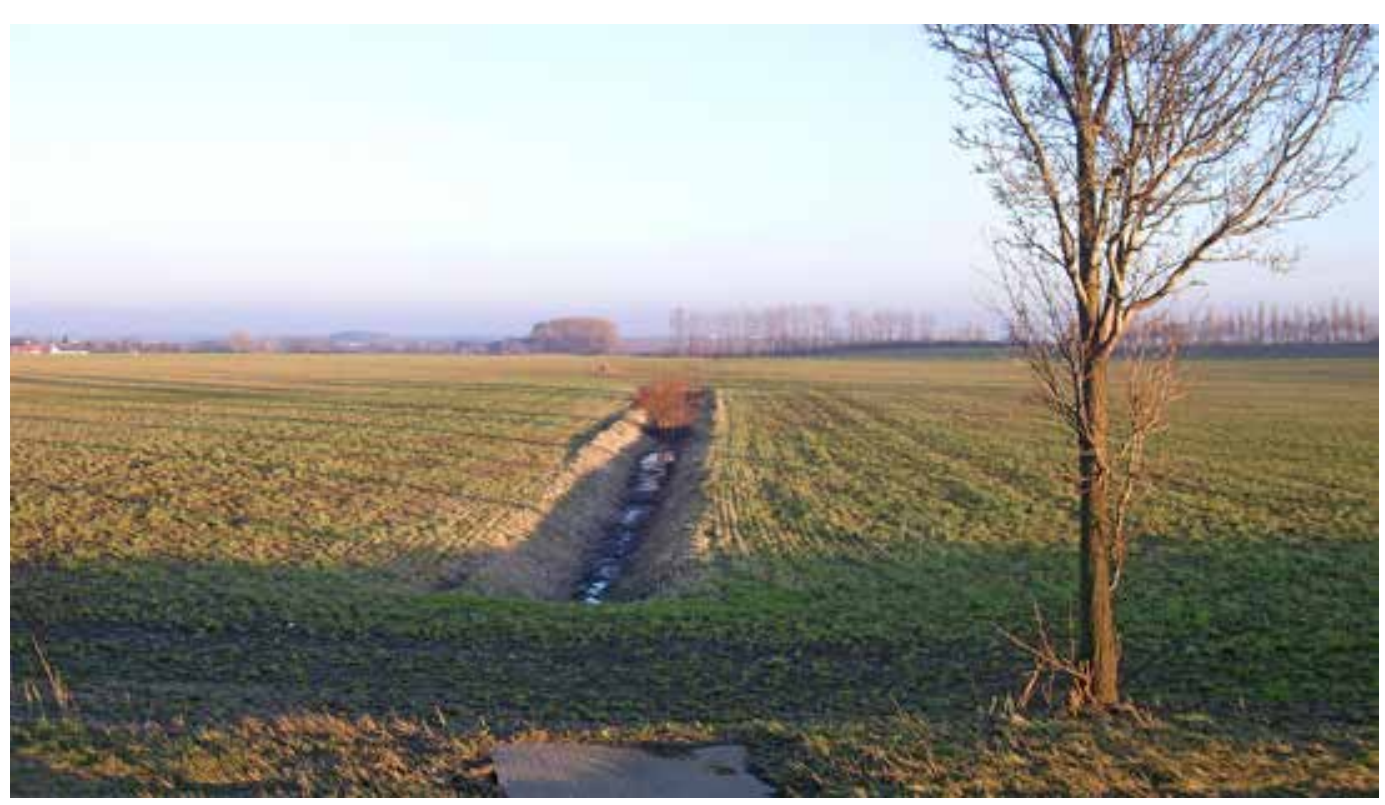


Frucht nicht versagt, erfüllt er, bedeckt mit Weizen, Hafer und Eicheln, die Gemüther der zahlreich an seinen Ufern zusammenströmenden Nachbarn mit froher Lust. Sobald aber wilde Kriegsläufe drohen, giebt er durch Blut und Asche gewisse Kunde der Zukunft. Diesen Quell verehrt und achtet daher jeder Eingeborene mehr als die Kirchen, wenn auch seine Vorzeichen trügerisch sind. Von ihm nun hat jener sich von der Elbe bis zum Flusse Chaminizi erstreckende Gau den Namen." ${ }^{15}$

Lommatzsch besaß damit keine typischen Voraussetzungen für die Entwicklung zur Stadt. Die anfangs zwar noch kleine Kirche war aber durch ihre erhöhte Lage in der Landschaft offenbar damals schon weithin sichtbar und bildete somit das rituelle Gegenstück zum heidnischen Kultplatz. Mit dem „modernen“ Patrozinium des Heiligen Wenzel, einem heiliggesprochenen Sohn eines böhmischen Herzogs ${ }^{16}$, ergaben sich für die Einwohner gewissermaßen Bezüge zur Herkunft ihres Stammes. Wanderten doch die Sorben des Gaues Daleminze im 7. Jahrhundert aus Böhmen ein. Die slawischen Einwohner erhielten mit dem Heiligen Wenzel folglich „einen von ihnen“ als Vertrauten und Patron ihrer Kirche, der es ihnen möglicherweise erleichtern sollte, die fremde Religion der Eroberer anzunehmen. ${ }^{17}$

Befand sich das Kirchlein anfangs noch einsam auf der Höhe, entstanden allmählich Wegebeziehungen von den Dörfern zum geistlichen Mittelpunkt. Die Siedlung Lommatzsch entwickelte sich mit der Zeit im Kreuzungspunkt von vier überregionalen Wegen. Diese führten nach Döbeln, Meißen, zum Elbübergang Merschwitz und zum Paltzschener See. ${ }^{18}$ Die Ostkolonisation von deutschen Bauern Anfang des 12. Jahrhunderts ließ die Bevölkerung sprunghaft ansteigen und Lommatzsch zur Stadt wachsen. Nach Blaschke soll ausgehend von der Kirche die Bebauung des Ortes erfolgt sein. Er unterscheidet nach Größe und Anlage der Grundstücke die Einwohner in Händler und Handwerker. Die Landwirtschaft spielte zur Stadtentstehung keine Rolle. Erst später sollen seiner Auffassung nach Bürger Ackerflächen erworben haben. Die Stadtfläche gab er mit 36 Hektar um die Kirche herum sehr klein an. ${ }^{19}$ Im Jahr 1286 wurde Lommatzsch als „civitas“ (Stadt) erstmals urkundlich erwähnt. ${ }^{20}$

Im 14. Jahrhundert muss es zu einem beträchtlichen Ausbau der Stadt gekommen sein. Dies bestätigen auch die aktuellen archäologischen Funde. Strobel und Spitzner fanden im Verlauf der Frauenstraße Hinweise auf einen spätmittelalterlichen Hohlweg, der von der Reißigstraße im Süden bis zur Einmündung des Unteren

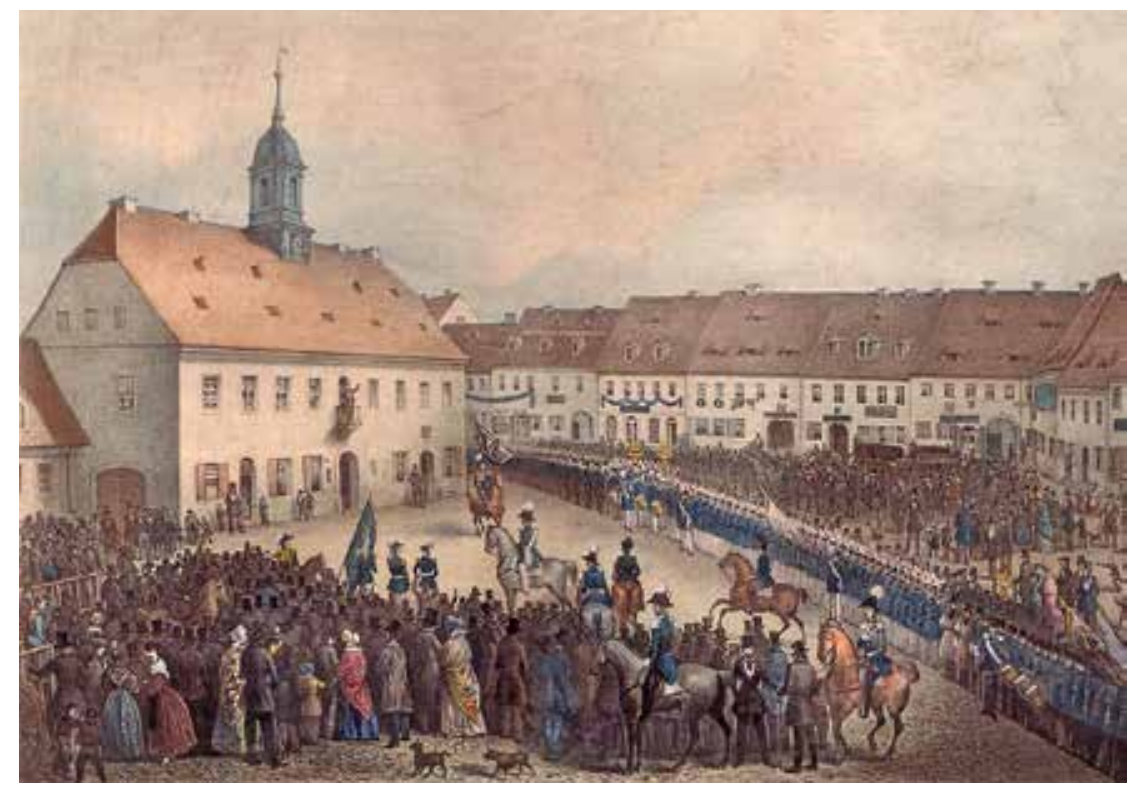

Marktes in die Meißner Straße führte. Ein Abzweig des Hohlweges ging über den Thossberg zur heutigen Döbelner Straße. Im Unteren Marktbereich (hinter dem heutigen Rathaus) gabelte sich der Hohlweg in Richtung der heutigen Döbelner und Meißner Straße. Im oberen Marktbereich fanden die Archäologen ebenfalls Rollierungen und Pflasterreste, die zu einer spätmittelalterlichen Straße gehörten. Vermutlich erfolgte die Aufgabe des Hohlweges im Bereich der heutigen Frauenstraße im Zuge des Ausbaus des Marktplatzes Ende des 14. Jahrhunderts, da dieser nun als offener Wassergraben etwa 9.000 Quadratmeter Fläche im Marktbereich entwässerte. Leder verarbeitendes Gewerbe sei aufgrund der Funde von Lederstücken im Bereich zwischen Frauengässchen und Mittlerem Korngässchen ansässig gewesen. ${ }^{21}$

Stadtbewohner, vor allem Handwerker, kamen in dieser Zeit vermutlich vorwiegend aus den umliegenden Dörfern. In der Stadt bildete sich eine arbeitsteilige Wirtschaft heraus. Das städtische Handwerk produzierte für die dörfliche Landwirtschaft, die Händler sorgten für den Warentausch regional wie überregional. Die Stadt gehörte dem Meißner Burggrafen, der als Stadtherr „kraft seiner Polizeiaufsicht über Handwerker, Verkaufsläden und Verkaufsbänken“ manche Einkünfte aus der Stadt bezog. 1330 bekam Burggraf Herrmann III. vom Markgrafen den Bierzins von Lommatzsch verliehen. Zudem unterstand ihm die Gerichtsbarkeit, Polizeigewalt und das Kirchenpatronat. ${ }^{22} 1348$ verlieh Burggraf Meinher IV. den Fleischern in Lommatzsch das Innungsrecht.

Die Christianisierung und der Aufbau des kirchlichen Lebens schienen stetig voran gegangen zu sein. Lommatzsch gehörte als Sitz eines Erz-
Der Lommatzscher Marktplatz zum Schützenfest 1845

๑ Sammlung Gerth Skibinski

meisters Karl Oskar Pilz sollen beim Stadtbrand 1734 umfangreiche Quellensammlungen, darunter die Stadthandelsbücher, verbrannt sein, vgl. Karl Oskar Pilz, Ein Bürgerbild aus alter Zeit, Lommatzsch 1883, S. 1.

6 Dietrich Zühlke: Elbtal und Lösshügelland bei Meißen. Ergebnisse der Heimatkundlichen Bestandsaufnahme in den Gebieten von Hirschstein und Meißen (Werte unserer Heimat, Bd. 32), Berlin 1979.

7 Vgl. Karlheinz Blaschke: Lommatzsch und Lausick. Zwei Kirchenstädte in Sachsen, in: Peter Johanek (Hrsg.): Stadtgrundriss und Stadtentwicklung. Forschungen zur Entstehung mitteleuropäischer Städte, Köln/Weimar/Wien 1997, S. 342-351.

8 Z.B. der Fund einer Votiveiner Tonfigur der Heiligen Barbara im Jahr 2009. Vgl. Spitzner/Strobel (wie Anm. 1), S. 186.

9 Vgl. Blaschke (wie Anm. 7), S. 342 .

10 Nach der letzten Gemeindefusion im Jahr 1994 gehören 37 Dörfer zur Stadt Lommatzsch. 1990 gehörten zur Stadt Lommatzsch die Dörfer Altlommatzsch, Jessen, Rauba, Scheerau, Schwochau, Pitschütz, ab 1994 kamen die ehemalige Gemeinde hand aus weißem Ton sowie 
Wuhnitz (Wuhnitz, Albertitz, Dennschütz, Marschütz, Weitzschenhain), die ehemalige Gemeinde Neckanitz (Neckanitz, Churschütz, Petzschwitz, Krepta, Birmenitz, Mögen, Poititz), die ehemalige Gemeinde Piskowitz (Piskowitz, Zscheilitz, Ickowitz, Löbschütz), die ehemalige Gemeinde Wachtnitz (Wachtnitz, Daubnitz, Zöthain, Prositz), die ehemalige Gemeinde Dörschnitz (Dörschnitz, Paltzschen, Sieglitz, Klappendorf, Lautzschen) und die ehemalige Gemeinde Striegnitz (Striegnitz, Barmenitz, Altsattel, Roitzsch, Trogen, Grauswitz) dazu. Einwohnerzahl (Stichtag 31.12.2016): 5.000 Einwohner.

11 Vgl. Karlheinz Blaschke: Geschichte Sachsens im Mittelalter, 2. Aufl. Berlin 1991, S. $24,29,35$.

12 Vgl. Blaschke (wie Anm. 11), S. 43-52; sowie Erich Keyser (Hrsg.): Deutsches Städtebuch, Berlin 1941, Bd. 2, S. 140 f.; vgl. zur namenskundlichen Herleitung der Bezeichnung Hainer Plaul: Über die frühe Geschichte der Lommatzscher Pflege, in: Lommatzscher Nachrichten, 1. Teil, Ausgabe 2 August 1991; 2. Teil, Ausgabe 1 September 1991; 3. Teil, Ausgabe 2 Dezember 1991; 4. Teil, Ausgabe 2 Januar 1992. Von den Deutschen sei der Gau Daleminizien genannt worden, von den Slawen Glomaci. Vom Wort Glomaci leitet sich der Stadtname Lommatzsch her.

13 Vgl. Blaschke (wie Anm. 7), S. 343 .

14 Zum Paltzschener See bzw. Glomatz-Brunnen vgl. Zahn (wie Anm. 5), S. 4-12.

15 Zitiert nach: Die Chronik des Thietmar von Merseburg, übersetzt von M. Laurent, 2. Auflage, durchgesehen und verbessert von Dr. I. Strebitzki, Leipzig 1879, S. 6 f.

16 Der böhmische Herzogssohn wurde wegen seines christlichen Glaubens 929 oder 935 ermordet und bald darauf heiliggesprochen. $\mathrm{Zu}-$ dem soll die Kirche mit einem „wunderthätigen Marienbild" ausgestattet worden sein. Vgl. Zahn (wie Anm. 4), S. 20; Blaschke (wie Anm. 7), S. 344.

17 Vgl. Blaschke (wie Anm. 7), S. 43 ff.; zum Heiligen St. Wen- priesters zur Propstei Meißen. Diesem Erzpriester unterstellt waren 17 Pfarrkirchen, darunter Striegnitz, Neckanitz und Dörschnitz. Die Lommatzscher Kirche besaß im 14. Jahrhundert drei Altäre, geweiht dem Heiligen Johannes, den Heiligen Fabian und Sebastian und der Jungfrau Maria. Zwei Bruderschaften - mit bischöflicher Genehmigung gegründete kirchliche Gemeinschaften - statteten ihren Mitgliedern feierliche Begräbnisse aus oder lasen Messen. ${ }^{23}$ Papst Innozenz VI. verlieh am 1. März 1359 der St. Wenzelskirche und dem darin befindlichen „wunderthätigen Marienbild“ einen Ablassbrief. ${ }^{24}$ Bis zum Ende des 15. Jahrhunderts wirkte Lommatzsch wohl noch als wichtiger Wallfahrtsort, denn den genannten Ablassbrief bestätigten 1461, 1474, 1477 und schließlich 1491 die jeweiligen Meißner Bischöfe. Als Wunder wurden dem Marienbilde und dem Heiligen Kreuz damals u. a. die Heilung von lahmen Knaben zugeschrieben. ${ }^{25}$

1408 verkaufte Burggraf Heinrich I. Lommatzsch mit allen Rechten, Zinsen, Gerichtseinkünften, Abgaben von der Ernte und drei zur Stadt gehörigen Vorwerken für $600 \mathrm{Gul-}$ den an die Markgrafen Friedrich IV. und Wilhelm II. von Meißen. ${ }^{26}$ Mit der unmittelbaren Unterstellung unter den Landesherrn besaß Lommatzsch zur damaligen Zeit die „höchste Stufe städtischer Autonomie“. ${ }^{27}$ Im 15. Jahrhundert führten alle wichtigen Zoll- und Handelsstraßen durch Lommatzsch. „Alle Wagen mit Gütern aus Markt Lausitz, aus Berlin, Stettin und anderen Orten sollen auf Herzberg, durch Hayn [Großenhain], auf Lommatzsch, Meißen, Dresden, Pirna und andere Gebirgsstädte durchfahren.“28

Die Verwaltung der Stadt lag in den Händen eines aus neun Personen zusammengesetzten Rates. Dieser war zugleich Schöffenkolleg des Stadtgerichts. Die oberste Gerichtsbarkeit übte der Landesherr aus.

Das Wachstum der Stadt vollzog sich nicht ohne Brüche. 1429 brachte der Hussitenkrieg Leid, Tote und Plünderungen. 1444 war die Stadt aufgrund der Brände für zwei Jahre von sämtlichen Abgaben und Diensten befreit worden. Fünf Jahre später brannte die Stadt im Krieg zwischen Kurfürst Friedrich II. und seinem Bruder Herzog Wilhelm III. von Sachsen erneut nieder. Auch in den folgenden Jahren kam es immer wieder zu Stadtbränden und Räubereien. ${ }^{29}$ Die Stadt Lommatzsch besaß damals keine Stadtmauern, weshalb kein ausreichender Schutz für Händler und Handwerker gegeben gewesen scheint. Zum Schutz der Bevölkerung dienten wohl vielfach die in Lehm gehauenen Keller unter Grundstücken im
Marktbereich. Deren eigentliche Bestimmung bestand jedoch aufgrund der ganzjährig gleichmäßigen Temperatur und Luftfeuchte in der Lagerung von Lebensmitteln, Bier und Feldfrüchten. ${ }^{30}$

1475 verlegte Herzog Albrecht der Beherzte von Sachsen den Jahrmarkt am Sonntag Judica aus Gründen der Sicherheit von Lommatzsch nach Meißen. Erst 1479 und bestätigt 1486 durch Herzog Albrecht den Beherzten durfte Lommatzsch einen neuen Jahrmarkt abhalten. ${ }^{31}$ Einen Wochenmarkt gab es jedoch trotzdem wöchentlich am Samstag. Lommatzsch zählte Ende des 15. Jahrhunderts weniger als 1.000 Einwohner. Aber Rückschläge boten zu jeder Zeit auch die Chance auf neues Wachstum.

\section{Neuzeit (16. bis 18. Jahrhundert)}

Mit dem Bau der noch heute das Stadtbild dominierenden Kirche setzte Lommatzsch zu Beginn des 16. Jahrhunderts ein Hoffnungszeichen. Brände und kriegerische Auseinandersetzungen in den Jahren zuvor hatten die alte Kirche stark in Mitleidenschaft gezogen. Mehrmals musste die Kirchgemeinde Geld für Reparaturen ausgeben. 1482 ließen sie die bei einem Brand geschmolzene Glocke neu gießen und 1494 den „Frauenaltar“ (gemeint ist der Marienaltar) restaurieren. 1504 begann der Kirchenumbau unter Leitung des Baumeisters Peter Ulrich von Pirna und begleitet von den Kirchenvorständen Georg Meusel und Barthel Möring. 1514 erfolgte die Kirchweihe. 1523 war der Bau bis auf den Innenausbau fertig gestellt. In den folgenden Jahrzehnten und Jahrhunderten wurde die Kirche mehrfach umgestaltet und ergänzt. Während die heute noch weithin sichtbaren drei Türme erst beim Umbau im 16. Jahrhundert hinzugefügt wurden, lässt sich das darunter stehende Turmsegment ins 12. Jahrhundert zurückdatieren. Als an Stelle des alten Langhauses der jetzige Hallenbau entstand, überragte der hohe Giebel wohl den Turm, weshalb das Obergeschoss um die dreispitzige Anlage ergänzt wurde. ${ }^{32}$ Die drei Türme sollen die heilige Dreifaltigkeit (Gott Vater, Gott Sohn und Gott Heiliger Geist) symbolisieren. Zu erwähnen sind hier außerdem die Anfertigung der Kanzel durch den Lommatzscher Tischlermeister Paul Steudte 1619 und der Ersatz des spätgotischen Altars der Kirche durch einen Barockaltar mit der Darstellung der Himmelfahrt Christi des Dresdner Bildhauers Paul Heermann von 1714.

Die vielen Stadtbrände waren ausschlaggebend für den Erlass von Vorschriften zur einheitlichen Bauweise im Stadtgebiet aus Stein. Die 
schlichten massiven Steinbauten besaßen zumeist nur ein Obergeschoss und nur selten Schlusssteine oder Verzierungen an den Toren. ${ }^{33}$ Nach der Anlage des Marktplatzes Ende des 14. Jahrhunderts erfolgte 1550 bis 1555 der Bau des Rathauses. Noch heute ist der auch damals schon vorhandene charakteristische Geländeabfall des „schiefen Marktes“ und seine einem unregelmäßigen Viereck gleichende Form zu erkennen. „Alte Abbildungen zeigen, daß der Giebel durch Schäfte aufgeteilt, also wohl in den Formen der Renaissance ausgebildet war. [...] an der Nordseite finden sich im Erdgeschoß 5 Fenster mit den Renaissanceprofilen der ersten Erbauungszeit.“34

Wie diese Baumaßnahmen zeigen, wuchs Lommatzsch im 16. Jahrhundert wieder. Damit einher gingen umfangreiche Veränderungen im Stadtbild, wie die aktuellen archäologischen Funde von Strobel und Spitzner im Abgleich mit der detaillierten zeitgenössischen Kartendarstellung von Matthias Oeder bestätigen. Die Verlegung des Friedhofes auf eine Wiese außerhalb der Stadt 1527/28, um in der Folge die Fläche von der Kirche bis zum Marktplatz städtisch entwickeln zu können, zeugt von einem größeren Platzbedarf und damit wieder mehr Einwohnern. Das 1540 von Markgraf Heinrich der Fromme verliehene „Bierprivilegium“, „kraft dessen innerhalb einer Meile um Lommatzsch herum nur Lommatzscher Bier verschenkt und vertrieben werden durfte“, würdigte mit den Worten „in Betrachtung ihres Nutzes und Zunehmens" die gute Entwicklung der Stadt. ${ }^{35} 14$ Jahre später bestätigt Kurfürst August von Sachsen „dem Städtlein Lommatzsch seine Statuta und Articul“, damit ge-

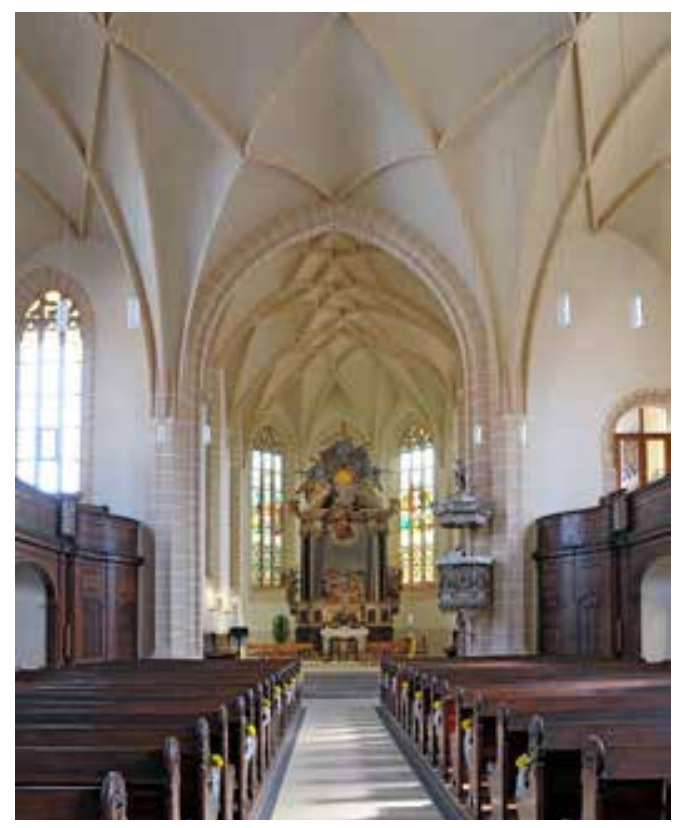

wissermaßen eine eigene "Gemeindeordnung“ für die Stadt. ${ }^{36}$ Diese regelte u. a. die Aufgaben des Stadtrates. Diesem oblag die Aufsicht u. a. über Handel und Gewerbe, den Bau von Häusern und die Obacht über die Stadthygiene wie die Abschaffung von „heimlich Gemach“ mit Ausgang auf die Gassen oder das Verbot, Blut und Schlachtabfälle durch die Fleischer auf die Gassen zu gießen. Niemand durfte ferner ohne Wissen des Rates sein „Erbe und Gütter, Häuser, Aecker, Wiesen, Flecke oder Scheunen“ teilen oder „außerhalb der Stadt an Frembde“ verkaufen. Damit nahm in Lommatzsch, wie für sächsische Städte typisch, die Entwicklung der städtischen Selbstverwaltung neue Züge an. ${ }^{37}$ Nach Katrin Keller breitete sich „der obrigkeitliche Charakter des Ratsregiments“ weiter aus. Die Landesherren griffen mit ihren Verfügungen einerseits unmittelbar in die städtische Organisation ein und integrierten die Städte damit zunehmend in staatliche Verwaltungsstrukturen. Andererseits übertrugen sie dabei den Räten wesentliche herrschaftliche Rechte gegenüber den übrigen Einwohnern der Stadt sowie eigene Gestaltungsräume für die innerstädtischen Angelegenheiten. Mit dem 1598 erstmals urkundlich gezeigten Wappen der Stadt Lommatzsch war nun auch die Würde und Bedeutung von Lommatzsch nach außen sichtbar.

War die frühe Neuzeit in Lommatzsch - wie in Sachsen generell - mit einem Aufschwung der Stadt verbunden, geriet der Ort im 17. Jahrhundert erneut in eine Krise. Für Lommatzsch wie Sachsen - fehlen intensivere Forschungen zur Stadtgeschichte in dieser Zeit, weshalb die Ursachen nur vermutet werden können. Louis Zahn beschreibt für Lommatzsch im Jahr 1607 zel vgl. auch die Recherchen: Auf den Spuren von St. Wenzel, Konfirmanden entdecken Lommatzscher Geschichte, MS, Lommatzsch 2010, S. 112 18 Vgl. Spitzner/Strobel (wie Anm. 1), S. 183: „Die ältesten Funde stammen aus einem Befund, der im Oberen Korngässchen angeschnitten wurde. Eine Grube, die in den anstehenden Lößlehm etwa $1 \mathrm{~m}$ eingetieft war, enthielt Keramik des 11./12. Jahrhunderts.“

19 Vgl. Blaschke (wie Anm. 7), S. 346 f. Die späteren Feldflächen pachteten oder kauften sie in den umliegenden Dörfern. Noch heute zeugt, das ehemals so genannte „Stadtgut" am Markt 18 mit seinem großen Hoftor vor der einstigen Nutzung als Wirtschaftshof in der Stadt. Vgl. Skibinski (wie Anm. 3), S. 15.

20 Vgl. Zahn (wie Anm. 5), S. 25. 21 Vgl. Spitzner/Strobel (wie Anm. 1), S. 186.

22 Vgl. Schmidt (wie Anm. 5), Sp. 962; Katrin Keller: Landesgeschichte Sachsen, Stuttgart 2002, S. 89 ff.; Blaschke (wie Anm. 7), S. 133.

23 Vgl. Schmidt (wie Anm. 5), Sp. 1000.

Links: Stadtkirche St. Wenzel in Lommatzsch, Innenansicht, Blick nach Osten

๑ Stadtverwaltung Lommatzsch

Rechts: Stadtkirche St. Wenzel in Lommatzsch, Postkarte, um 1900

๑) Sammlung Gerth Skibinski

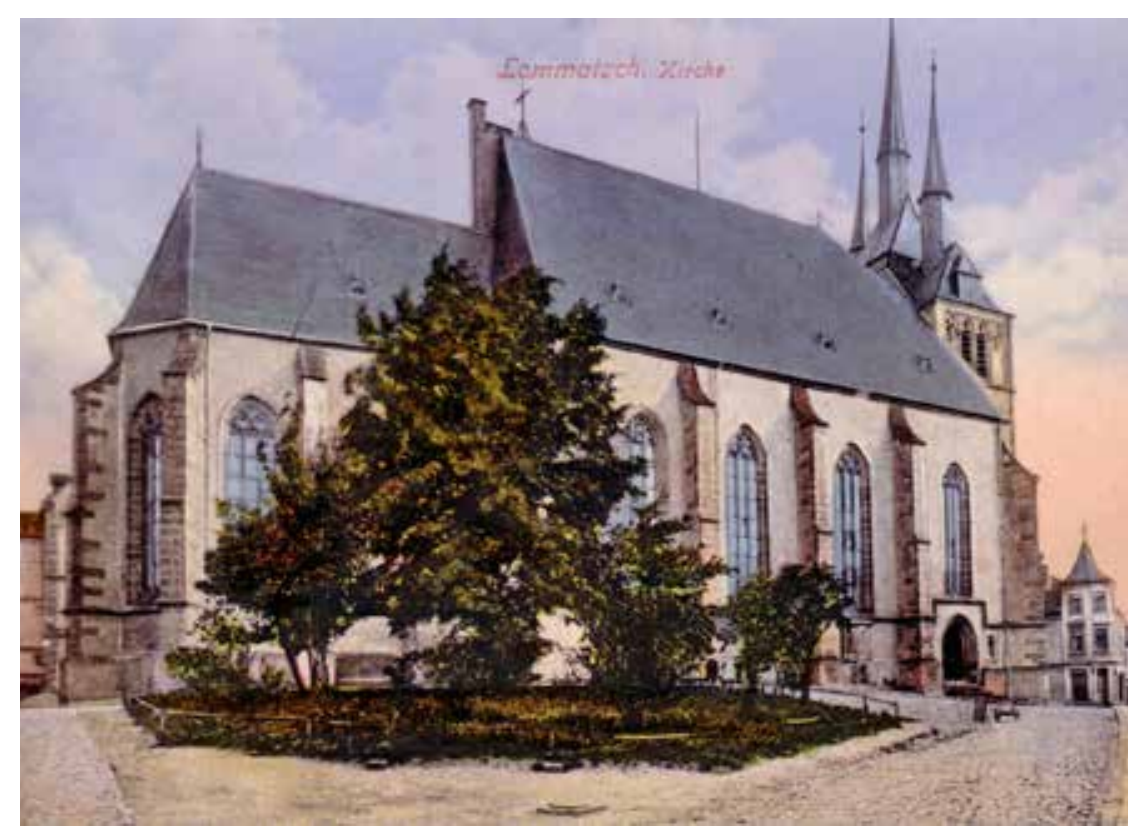




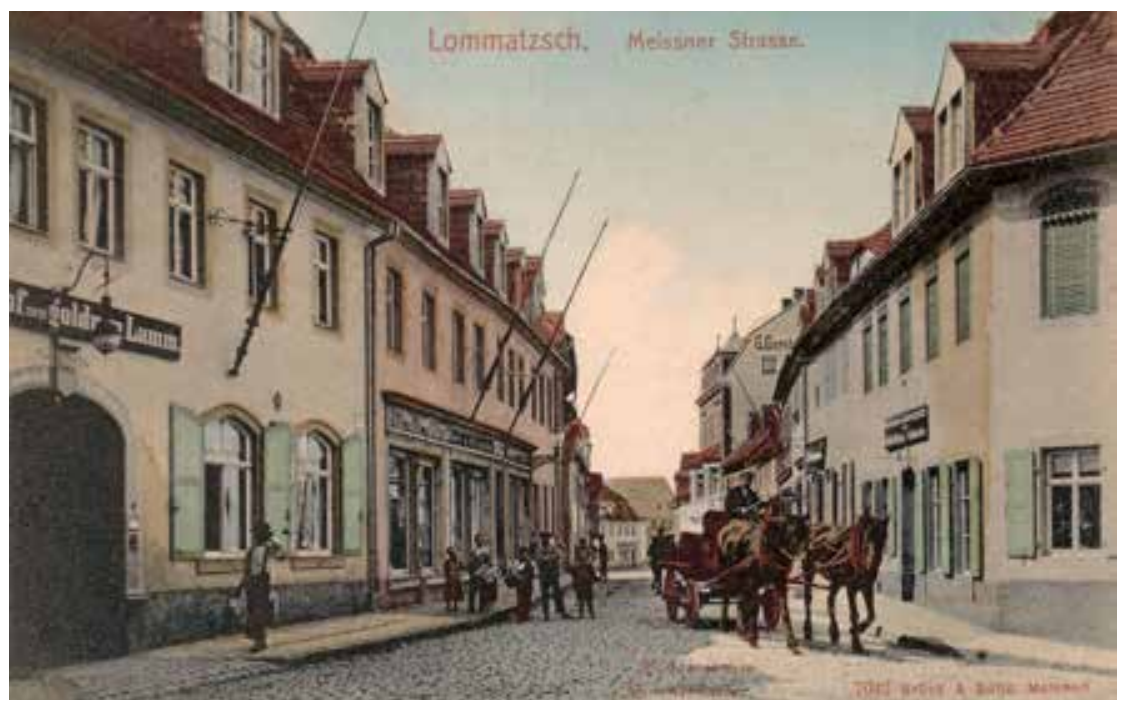

Meißner Straße in Lommatzsch, Postkarte, um 1900 ๑ Sammlung Gerth Skibinski

24 Vgl. Ebenda sowie Zahn (wie Anm. 5), S. 27.

25 Vgl. Zahn (wie Anm. 5), S. 32 sowie zum Wallfahrtsort vgl. Spitzner/Strobel (wie Anm. 1), S. 185 ff.

26 Schmidt (wie Anm. 5), Sp. 996.

27 Blaschke (wie Anm. 7), S. 347.

28 Zitiert nach Schmidt (wie Anm. 5), Sp. 996, neue Straßen- und Zollordnung des Kurfürsten Friedrich des Sanftmütigen vom Jahre 1462. Nach Blaschke gab es in der Stadt 4 Stadttore an den wichtigsten Straßen, das Strehlaer, Döbelner, Großenhainer und Meißner Tor. Für seine Theorie des Ochsenhandels durch die Stadt fanden die Archäologen rings um das Rathaus noch keine Belege. Die von Blaschke zitierte „Hornstraße“ ist vermutlich fälschlicherweise von ihm so genannt worden, da es sich auf den Karten um die noch heute so bezeichnete „Kornstraße“ handelt. Die "Ochsenstraße“ von Lommatzsch in Richtung Elbe gibt es als Feld- und Radweg noch heute. Vgl. Blaschke (wie Anm. 7), S. $346 \mathrm{f}$.

29 Vgl. Zahn (wie Anm. 5), S. 3336; Siegfried Börtitz/Herbert Grund: Die mittelalterlichen Keller im Stadtgebiet von Lommatzsch, in: Sächsische Heimatblätter 18 (1972), S. 105-110, hier S. 106.

Stadtgut am Markt, 1905 ( ) Sammlung Gerth Skibinski ein „großes Sterben“, dem über „elfhundert Personen“ in der Stadt, „mehrenteils alte Leute“, erlagen. ${ }^{38}$ Die Pest, die vermutlich hierfür die Ursache war, grassierte zwischen 1611 und 1633 mehrfach in der Stadt und in benachbarten Dörfern. Obwohl Lommatzsch zwischen 1560 und 1600 Zuzug erlebte und der Sterbeüberhang geringer als die Geburtenzahl war, wird die Anzahl der Einwohner zum 31. Dezember 1600 laut den Angaben der „Neuen Sächsischen Kirchengalerie" in etwa 1.000 Köpfe betragen haben. 1697 soll Lommatzsch 771 und drei Jahre später ca. 800 Einwohner gehabt haben. Demnach war die Einwohnerzahl etwa ähnlich hoch wie im Jahr $1500 .{ }^{39}$ Der Dreißigjährige Krieg verschonte auch Lommatzsch nicht. 1637 wurde die Stadt „bis auf die Kirche, Schule und Rathaus in Asche gelegt“" ${ }^{40} 1642$ plünderten und wüteten die durchziehenden Truppen der Schweden in der Stadt. Zum Schutz bildete die Bürgerschaft eine Bürgerwehr, die 1648 im Stadthandelsbuch erwähnt wird. ${ }^{41}$ Urkundlich belegt ist im Jahr 1688 auch die erste bekannte natürliche Geburt von Fünflingen in Sachsen. Noch heute erinnert der Grabstein für die Familie Kühne in der Brautstube der St. Wenzelskirche an dieses Ereignis. ${ }^{42}$

Bis zum Ende des 17. Jahrhunderts erlebte Lommatzsch keine Blüte mehr. Unwetter, Dürren, Krankheiten und Brände machten den Menschen das Leben schwer. Das Rückgrat der Stadt waren die Handwerker, die immer wieder für den städtischen Wiederaufbau sorgten und die Versorgung der städtischen Bevölkerung sicher stellten Nach den Aufzeichnungen von Louis Zahn lebten 1697 in Lommatzsch „11 Handelsleute, 102 brauende Bürger, 6 Bäcker, 9 Fleischer, 5 Tuch- und Zeuchmacher, 10 Leineweber [...] und 100 andere Handwerker. Man braute jährlich gegen $1100 \mathrm{Faß}$ Bier und hielt
26 Pferde und 174 Kühe." ${ }^{43}$ Damals gab es 236 bewohnte und 52 unbewohnte Häuser, wovon 45 Häuser schon seit 1632 verfallen gewesen sein sollen. Damit unterschied sich Lommatzsch nicht von anderen sächsischen Städten, sollen doch Ende des 17./Anfang des 18. Jahrhundert in einem Drittel aller sächsischen Städte ruinöse Häuser aus den Zeiten des Dreißigjähren Krieges gestanden haben. Am schnellsten erholten sich wohl die Orte, die vor allem die Funktion der Nahversorgung für das unmittelbare Umland besaßen, dadurch auch in Kriegszeiten gebraucht wurden und Handelsplätze für Güter blieben. Für Lommatzsch ist eine solche Funktion zu erwarten, da der Wiederaufbau z. B. nach den Stadtbränden 1727 und 1734, denen viele Häuser, Scheunen und das Rathaus zum Opfer fielen, vergleichsweise schnell ging. Bereits 1738 war das Rathaus nun im barocken Stil wieder aufgebaut und mit neuen architektonischen Merkmalen wie dem Rathausturm ausgestattet. ${ }^{44}$ Zwischen 1700 und 1900 soll sich die Einwohnerzahl vervierfacht haben, wobei das 19. Jahrhundert das größte Bevölkerungswachstum auswies. Gab es im Jahr 1800 bereits 1.261 Einwohner, zählte die Stadt am Ende des Jahrhunderts rund 3.200. ${ }^{45}$

Zur Pfarrkirche Lommatzsch gehören die Dörfer Altlommatzsch, Sieglitz, Scheerau, Pitschütz, Jessen, Löbschütz, Paltzschen, Lautzschen, Prositz, Zscheilitz, Zöthain, Rauba, Messa, Domselwitz, Wachtnitz und Daubnitz.

Nach den überstandenen Bränden Anfang des 18. Jahrhunderts belastete der Siebenjährige Krieg mit häufigen privaten Einquartierungen von Soldaten sowie die Abordnung von Rekruten aus der Lommatzscher Bevölkerung für die preußische Armee die Stadt schwer. ${ }^{46} 1760$ diente die St. Wenzelskirche der preußischen Armee als Getreidelager, wofür Emporen und Kirchengestühl weichen mussten. Trotz der hohen finanziellen Belastungen erfolgte nach Friedensschluss ein rascher Wiederaufbau der Kirche mit neuer Emporenanlage 1767 und

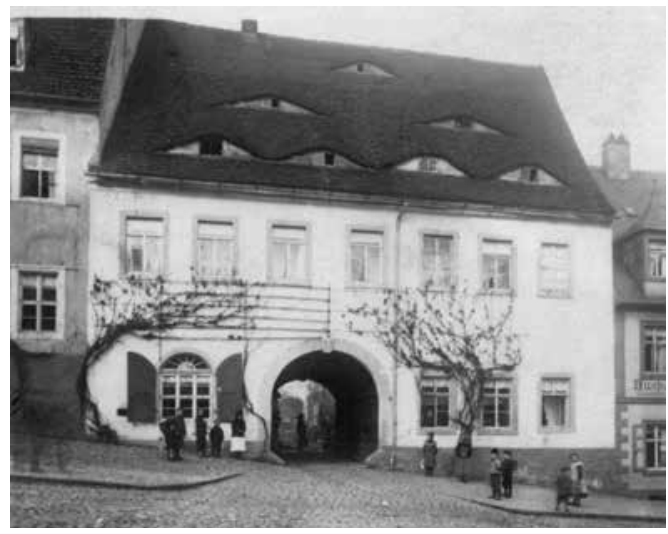


Orgelreparatur. ${ }^{47}$ Ende des 18. Jahrhunderts bestimmten weiterhin die Handwerker die Lommatzscher Gewerbelandschaft, wobei sich die Professionen zunehmend spezialisierten. In Lommatzsch gewann sogar die Strumpfwirkerei an Bedeutung, die gemeinhin eher dem Chemnitzer Umland zugeordnet wird. Einerseits sollen die Lommatzscher Bauern in Größenordnung den Rauh- und Weberkardenanbau betrieben haben. Die Distelpflanze diente zur Glättung von Wollfäden in der frühen Textilindustrie. Andererseits produzierten Lommatzscher Handwerker Anfang des 19. Jahrhunderts laut den Aufzeichnungen von Louis Zahn „700 Duzend wollene Strümpfe und Handschuhe, gegen 1400 Schocke Web-Leinwand, 2400 Stück Hüte“. Auch das Gerber- und Töpferhandwerk waren verbreitet und es gab wohl eine kleine „Tabakfabrik“48. Die Stadt erholte sich langsam wieder. Doch zu Beginn des 19. Jahrhunderts verlangten die Kriegsjahre 1813/14 der Stadt und den umliegenden Dörfern erneut Einquartierungen und Versorgungsleistungen für die durchziehenden Truppen ab. Damit endete die Neuzeit gewissermaßen, wie sie begonnen hatte, mit Leid und Tod.

Eine historische Zäsur und damit zugleich der Startpunkt in das industrielle Zeitalter mit den dadurch möglichen Veränderungen der landwirtschaftlichen Produktion ist für Lommatzsch mit dem Beginn der Agrarreformen in Sachsen 1832 zu setzen. Der gewerblich-industrielle Aufschwung in der Stadt selbst ließ jedoch noch fast ein halbes Jahrhundert länger auf sich warten.

\section{Industrialisierung (19./20. Jahrhundert)}

Nach den Recherchen von Gernot Härtner wies Lommatzsch zu Beginn des 19. Jahrhunderts den Charakter einer Ackerbürgerstadt auf. ${ }^{49}$ Die Bewohner betrieben neben ihrem Handwerk auch Feldwirtschaft und Viehhaltung. Auf jede Familie seien 900 Quadratmeter Land gekommen. In fast jedem Haus sollen Hühner gehalten worden sein, dazu kamen rund 1.200 Nutztiere in der Stadt wie Kühe, Pferde bis hin zu Ziegen. Im sogenannten Stadtgut, dem Gebäude Markt 18 in Lommatzsch, hielt sich die landwirtschaftliche Nutzung im Stadtzentrum noch bis ins 20 . Jahrhundert. Die Kühe wurden gleich hinter dem Gut auf die Wiese getrieben. Härtner gab zudem 70 verschiedene Handwerke und Gewerbe in der Stadt an, aber noch keine Industrie.

So wie die städtische Wirtschaftsstruktur mit ihren Handwerkern und Landwirten im Nebenerwerb bis zum Ende des 19. Jahrhunderts wirtschaftlich eher rückständig erschien, war auch die städtische Infrastruktur unzeitgemäß. 1858 beklagten die Mitglieder des Gewerbevereins im „Lommatzscher Anzeiger“: „Der Mangel an Wasser und besonders an gutem Wasser ist seit längerer Zeit und oft von den Einwohnern hiesiger Stadt beklagt worden [...]. wie kommt es, daß in einer Stadt, welche sich doch auch zu den gebildeten und intelligenten zählt und unter die wohlhabenden zur rechnen ist, in diesem so nahe liegenden menschlichen Bedürfnisse Verbesserungen nur gewünscht, aber nicht gemacht worden sind?" 50

Tatsächlich gab es schon im 16. Jahrhundert eine hölzerne Wasserleitung, die Wasser von Altlommatzsch zum Bierbrauen in die Stadt leitete. Als tägliches Brauchwasser nahmen die Hausfrauen jedoch Brunnenwasser, welches sehr kalkhaltig war und offenbar nicht schmeckte. ${ }^{51}$ Teilweise fingen sie deshalb für die Wäsche das wesentlich weichere Regenwasser auf. In der Amtszeit des Bürgermeisters Louis Zahn beschlossen Stadtverordnetenkollegium und Stadtrat 1891, eine Wasserleitung von Churschütz nach Lommatzsch zu bauen. 1894 waren 340 Haushalte - Lommatzsch hatte damals 2.971 Einwohner - an die Wasserleitung angeschlossen. Finanziert wurde diese über Kredite, über einen Teil der Gewinne der städtischen Sparkasse und über eine private Schenkung. 1898 erhielt der Bahnhof einen Wasseranschluss zur Befüllung der Lokomotiven. ${ }^{52}$
30 Vgl. Börtitz/Grund (wie Anm. 29), S. 107. Sie verweisen auf die wohl älteste chronikalische Nachricht zur Existenz und Verwendung der Keller in der Chronik von Valentin Lossius im Jahr 1629.

31 Vgl. Zahn (wie Anm. 5), S. 34 f. 32 Vgl. Gurlitt (wie Anm. 5), S. 269-275, dort umfangreiche Darstellung der Baugeschichte. Vgl. auch: Dieter Keil: Baugeschichte der St. Wenzel-Kirche, ungedr. MS 2017, Privatarchiv Keil. Die Namenszüge von Georg Meusel und seiner Frau sind noch immer im Kirchenschiff, an der südlichen Fensterfront sichtbar und sollten wahrscheinlich allezeit an seine damalige Rolle erinnern. Dreitürmige Kirchen sind selten in Deutschland. Drei ähnliche Turmbauten besitzen die Bethlehemskirche in Hannover-Linden, die Severinkirche in Erfurt und die Stadtkirche in Oederan.

33 Vgl. Gurlitt (wie Anm. 5), S. 295.

34 Ebenda, S. 295.

35 Vgl. Zahn (wie Anm. 5), S. 40. Im Jahr 1528 kaufte die Kirche eine Wiese auf der Gemarkung Messa, um in der Folge den bisher nördlich neben der Kirche gelege-

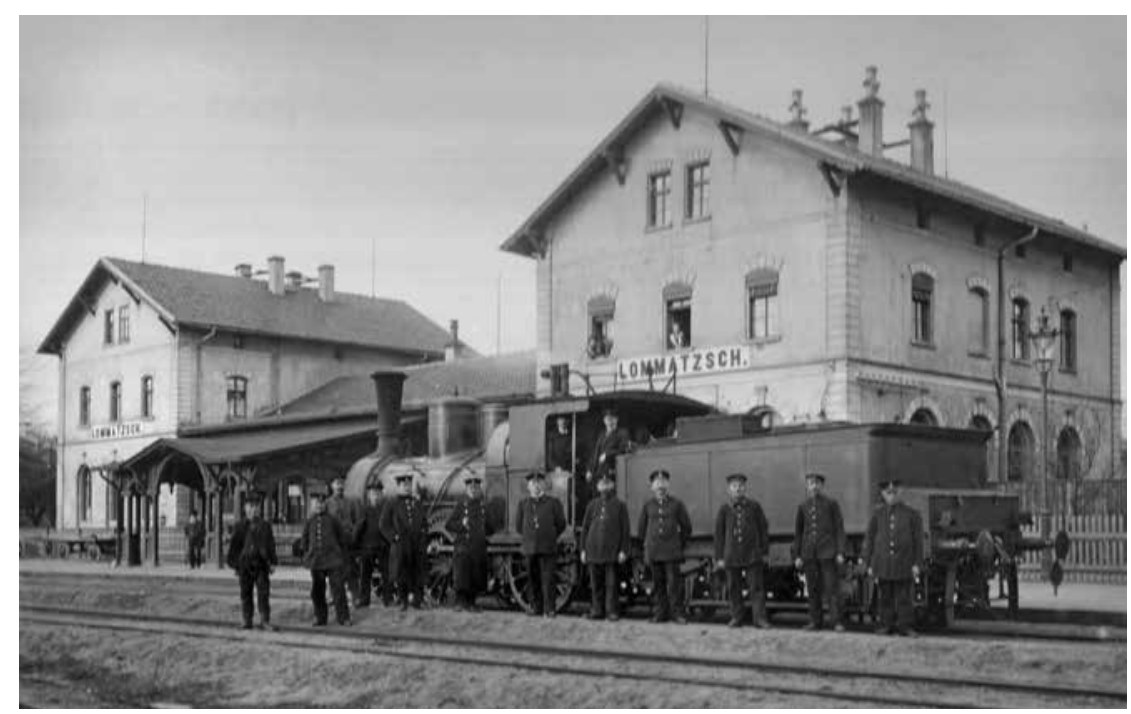

Mit dem Bau der Eisenbahnlinie Lommatzsch - Riesa erhoffte sich die Stadt Lommatzsch, am industriellen Aufschwung in Sachsen teilhaben zu können. Riesa profierte bereits seit 1839 vom Bahnanschluss an die Bahnlinie Leipzig Dresden. Damals kam Lommatzsch im Rahmen der Diskussion über die Trassenführung nicht zum Zuge. Als 1865 der Bau der Eisenbahn von
Bahnhof Lommatzsch mit Bahnhofsvorsteher und Personal, um 1910

๑) Sammlung Gerth Skibinski 
Glasfabrik Carl Menzel und Söhne (Carlswerk), 1911 ๑) Sammlung Gerth Skibinski nen Friedhof dorthin zu verlegen.

36 Vgl. Zahn (wie Anm. 5), S. 47. 37 Keller (wie Anm. 22), S. 192.

38 Vgl. Zahn (wie Anm. 5), S. 57 ff. Wobei sich die Anzahl nicht allein durch Einwohner erklären lässt, da die Stadt sonst keine Einwohner mehr gehabt hätte, sondern vielleicht auch durch Flüchtlinge aus den umliegenden Dörfern und Städten, in denen vermutlich ebenfalls die Pest grassierte.

39 Vgl. Schmidt (wie Anm. 5), Sp. 1018.

40 Vgl. Zahn (wie Anm. 5), S. $67 \mathrm{ff}$.

41 Vgl. Pilz (wie Anm. 5),, S. 4 f. mit einem Auszug aus dem 7. Stadthandelsbuch.

42 Vgl. Ekkehard Paditz: „Fünff Kinder auf ein mahl begrüsten diese Welt". Fünflinge in Lommatzsch im Jahre 1688. Historischer Beitrag zur Mehrlingsforschung und Lebenserwartung im 17./18. Jahrhundert, hrsg. anlässlich des 2. Symposiums über Neugeborenen-Intensivtherapie, Dresden 1988. Nach Paditz waren Kinderzahlen zwischen 4 und 5 im 17./18. Jahrhundert die Regel, wobei die Überlebenszahl aufgrund der hohen Säuglings- und Kindersterblichkeit deutlich geringer war. Für 1732 ermittelte Paditz 32,8 Prozent der Todesfälle von Kindern/ Jugendliche bis zum 18. Lebensjahr, wobei 25 Prozent bis zum 5. Lebensjahr starben. 10 Prozent der Sterbefälle waren Personen im Alter von 19 bis 40 Jahren und eine deutliche Zunahme gab es an Verstorbenen in der Gruppe der über 70-jährigen. Damit lag Anfang des 18. Jahrhunderts die tatsächliche Lebenserwartung in Lommatzsch deutlich höher als die durchschnittlich ermittelte Lebenserwartung von 39 Jahren. Über 70-Jährige waren keine Seltenheit.

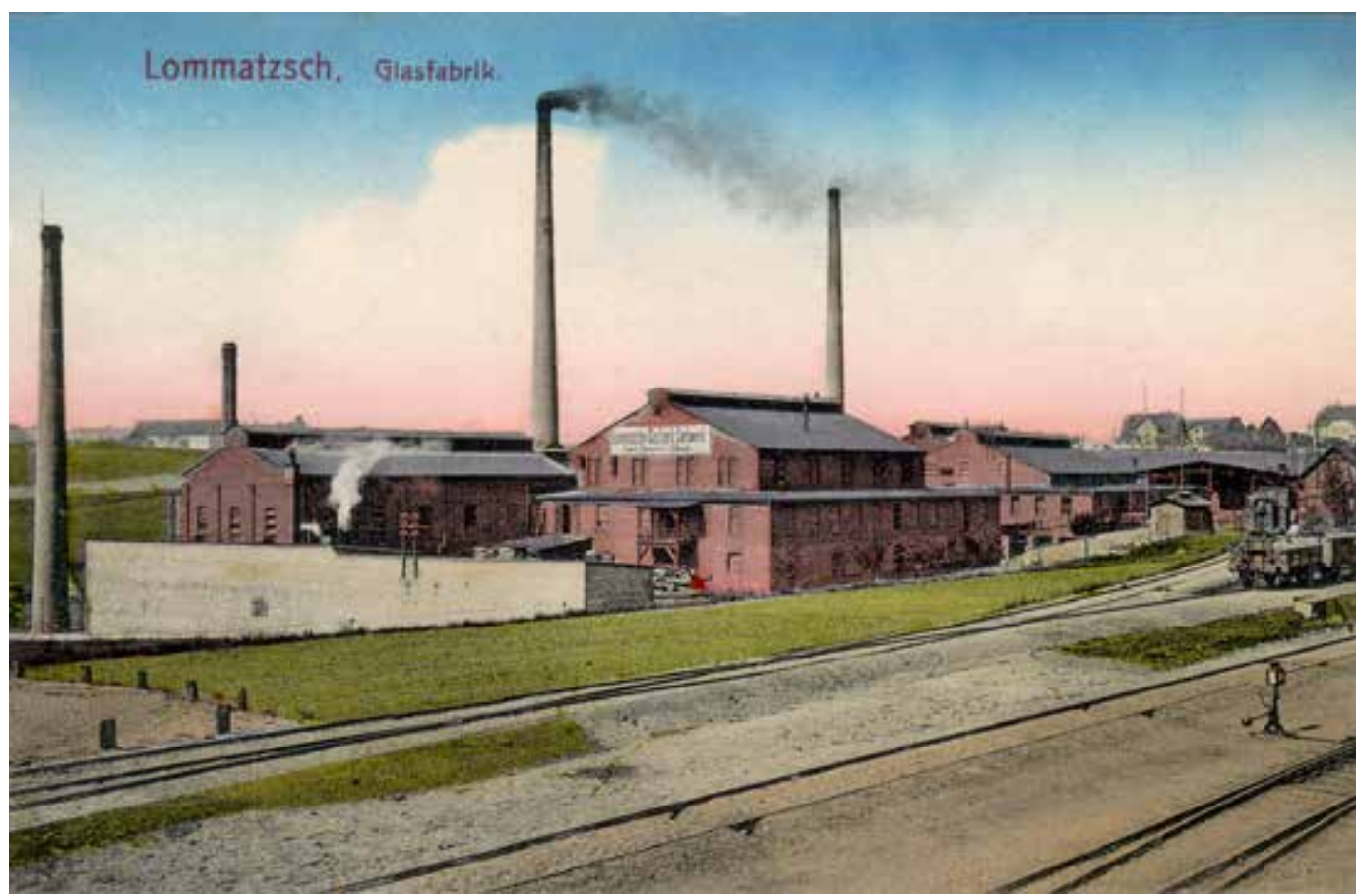

Leipzig nach Dresden über Döbeln im Bau war, regte der Apotheker Friedrich Wilhelm Herb auch den Anschluss von Lommatzsch ans Bahnnetz an. Erst nachdem sich die Bürgermeister von Nossen, Lommatzsch und Riesa gemeinsam für den Bau einer Bahnlinie stark machten, erfolgte schließlich 1875 der Spatenstich. ${ }^{53}$ Mit dem Bau der Eisenbahnlinie von Riesa über Lommatzsch nach Nossen und Anschluss nach Freiberg erhofften sich die Unterstützer, die verkehrsmäßig ungünstige Lage der Stadt für die wirtschaftliche Entwicklung auszugleichen. Trotzdem sollte es noch gut 20 Jahre dauern, bis 1897 Carl Menzel mit einer Glasfabrik die industrielle Entwicklung der Stadt beförderte und damit genau den Vorteil des Bahnanschlusses zu nutzen wusste. Zwar legte mit Moritz Buschmann 1861 ein Handwerksmeister ebenso den Grundstein für spätere Betriebe im Metall- und Gerätebau, auf deren Wurzeln sich heute noch drei existierende Betriebe berufen. ${ }^{54}$ Doch beschäftigte Buschmann im Vergleich zum Glaswerk Carl Menzel und Söhne nur ein Zehntel der Arbeitskräfte. Anfänglich sollen im Glaswerk 200 Arbeitskräfte tätig gewesen sein. Diese warb der Betrieb zum Teil vor Ort, aber auch in Böhmen und Ungarn an. Die eigentliche Entwicklung von Industriebetrieben begann aber erst Anfang des 20. Jahrhunderts, südlich und nördlich des Bahnhofes. 1919 gründete sich die Firma Gotthardt \& Kühne, die sich zu einem bedeutenden Betrieb für Futterdämpferanlagen entwickelte und in den 1950er Jahren rund 300 Mitarbeiter beschäftigte. Zwei ehemalige Glas- bläser, Bruno Lehmann und Paul Balzer, machten sich 1926 mit der Herstellung von Spiegeln selbständig. Diese Firma entwickelte sich zum leistungsstärksten Betrieb der Glasveredelung in Lommatzsch und war als VEB Glastechnik in der DDR ab 1984 Alleinhersteller von Automobil- und Straßenverkehrsspiegeln.

Ebenfalls 1919 gründeten 36 Groß- und Mittelbauern die Lommatzscher Gemüse und ObstVerwertungs-GmbH. Diese bildete das Fundament der heutigen Elbtal Tiefkühlkost GmbH, einem Betriebsteil der Frosta AG. Sie erkannten damals die Notwendigkeit einer „Zentralstelle für den Absatz und die Verwertung der in der Lommatzscher Pflege angebauten Gemüseund Obstarten [...], um alle im reichen Maß anfallenden Rohwaren verlustlos der menschlichen Ernährung zuzuführen. " ${ }^{55}$ Die Herstellung und der Vertrieb von Gemüse- und Obstkonserven, Marmelade, Sauerkraut und der Vertrieb von Frischware bildeten das Geschäftsfeld. Auch dieser Betrieb siedelte sich unmittelbar an der Bahnstrecke Riesa-Lommatzsch, allerdings auf Messaer Flur, an und bekam einen eigenen Gleisanschluss gelegt. In den 1930er Jahren waren in der Saison teilweise bis zu 300 Frauen beschäftigt.

Die industrielle Weiterverarbeitung von Rohwaren der Felder direkt am Ort der Erzeugung verweist nicht nur auf den großen Lebensmittelbedarf im Gründungsjahr, sondern unterstreicht den Wandel der Landwirtschaft seit dem Beginn des 19. Jahrhunderts. War bis 1830 die Landwirtschaft bestimmend für die wirtschaftliche und soziale Entwicklung von 
Sachsen gewesen, verlor diese nun an gesamtökonomischer Bedeutung. ${ }^{56}$ Sachsen besaß innerhalb des Deutschen Reiches die dichteste Besiedelung und zugleich den geringsten Anteil an landwirtschaftlicher Bevölkerung. Dabei vollzog sich die industrielle Entwicklung jedoch in enger Wechselwirkung mit einer Intensivierung der Landwirtschaft. Für wirtschaftliche und technische Neuerungen in diesem Wirtschaftszweig bildete die Agrarreform in Sachsen die Voraussetzung. Da die sächsischen Bauern im Gegensatz zu den preußischen nicht unter Leibeigenschaft litten, setzte die Reform der landwirtschaftlichen Rechtsverhältnisse spät ein. Auf Grundlage des Gesetzes über Ablösungen und Gemeinheitsteilungen 1832 und der dazu folgenden Nachtragsgesetze gelang es bis zum Jahr 1859, alle feudalen Lasten der Bauern (z. B. Naturalabgaben, Frondienst-, Lehngeld- und Hutungsablösungen) mittels Geldzahlungen abzulösen. Daneben gab es weitere Reformen beispielsweise zu Grundstückszusammenlegungen, zur Reorganisation des Hypothekenwesens und zur Grundsteuer. ${ }^{57}$ Diese Reformen stärkten vor allem die mittelgroßen Bauern. ${ }^{58}$ Die Ablösung erfolgte in Geld, nicht in Landabtretungen. Diese sowie die Anwendung künstlicher Düngemittel und der Einzug von technischen landwirtschaftlichen Maschinen ließen die landwirtschaftlichen Erträge steigen. Die Getreideproduktion nahm zu, der Viehbestand vergrößerte sich und der Landbesitz wurde erweitert. In der Lommatzscher Pflege zeugen heute noch die großen Drei- und Vierseithöfe mit den auf den Schlusssteinen verewigten Bauzeiten zumeist Mitte bis Ende des 19. Jahrhunderts von dieser Blütezeit. Nach einem Artikel aus dem Jahr 1935 hielten die Maschinen in der Landwirtschaft der Lommatzscher Pflege etwa in den 1880er Jahren Einzug. ${ }^{59}$ Mit dem Bau der Kleinbahnen nach Meißen und Döbeln (1909/10) verbesserten sich auch die Transportbeziehungen in und aus der Lommatzscher Pflege vor allem für Getreide, Zuckerrüben, Gemüse, Milch, aber auch für Düngemittel, Löthainer Rohkaolin und Käbschützer Steine entscheidend. ${ }^{60}$ Im Volksmund gilt diese Bahn bis heute auch als „Zuckerrübenbahn“.

Der Erste und Zweite Weltkrieg brachten für Lommatzsch weitreichende Einschnitte und Veränderungen. Gottfried Ostermay fasste die Ereignisse mit den Worten zusammen: „Durch den zweiten Weltkrieg wurde das Leben unserer Stadt und ihrer Einwohner stark in Mitleidenschaft gezogen. Die Tage Ende April und Anfang Mai 1945 brachten uns den Wahnsinn eines Krieges deutlich vor Augen."61 Die Ver- sorgung der Einwohner und der vielen Flüchtlinge bzw. „Umsiedler“ sowie deren zumindest behelfsmäßiger Unterbringung blieb in den ersten Nachkriegsjahren schwierig. Im Zuge der Bodenreform wurden Güter u.a. in Jessen, Petzschwitz und Schleinitz enteignet, aufgeteilt und an ehemalige Landarbeiter und Flüchtlinge als „Neubauernstellen zu etwa 5 h“ übergeben. ${ }^{62}$ Begleiterscheinung der Bodenreform war eine Ernährungskrise in Sachsen 1946/47. „Die Art und Weise der Durchführung belastete nachhaltig den antifaschistischen Grundkonsens, der den Beginn des Wiederaufbaus in der Sowjetischen Besatzungszone geprägt hatte. Die soziale Struktur der sächsischen Dörfer und die Prägung der Landwirtschaft hatte sie freilich nicht grundlegend geändert: bis zur Kollektivierung der 50er-Jahre blieb sie von Klein- und Mittelbauern dominiert.“" ${ }^{33}$ Ab 1952 begann in der Lommatzscher Pflege die Gründung von Landwirtschaftlichen Produktionsgenossenschaften (LPG) mit 50 bis 300 Hektar Größe, ab 1960 die „Vollgenossenschaften“.64 Die sozialistische Umgestaltung der Landwirtschaft mit politisch vorgegebenen Zielen zur Ertragssteigerung brachte weitere Spezialisierungen und Kooperationen mit sich. 1969 wurde das Trockenwerk in Lommatzsch gegründet (heute befindet sich die Firma K \& K Sondermaschinen und Förderanlagen auf dem Betriebsgelände), die ZGE Frischeier Schwochau (heute Geflügelfarm Schwochau $\mathrm{GmbH}$ ) und 1972 das Agrochemische Zentrum (nach 1991 als TDG GmbH privatisiert und 2017 an die Firma Beiselen verkauft). Zudem gewann die kommunale Zusammenarbeit an Bedeutung. 1972 gründete sich der Gemeindeverband Lommatzsch, dem neben der Stadt Lommatzsch sieben Gemeinden mit 48 Ortsteilen angehörten. ${ }^{65}$

Die Wohnbedingungen in der Stadt verbesserten sich im Laufe der DDR-Zeit wesentlich. Durch staatlichen, genossenschaftlichen sowie LPG-Geschossbau und den Bau von 45 Eigenheimen entstanden 411 Wohnungen bis 1986. Das Stadtbild veränderte sich, war nun von der Robert-Volkmann-Allee bis zum Bahnhof bebaut. Neben der 1859 gebauten „alten Schule“ wurde 1974 die „neue Schule“ als Plattenbau „Typ Dresden“ errichtet. Kindertagesstätten wurden nach 1945 eingerichtet und bis zum Ende der DDR stetig weiterentwickelt. Neben der Landwirtschaft brauchten die Lommatzscher Betriebe Arbeitskräfte. Für diese gab es ein reges Vereins- und Kulturleben. Die für die Stadt Lommatzsch bezogen auf ihr Wachsen positive Entwicklung der DDR-Zeit endete mit der Friedlichen Revolution 1989/90.
43 Vgl. Zahn (wie Anm. 5), S. 85; Keller (wie Anm. 22), S. 194.

44 Vgl. Keller (wie Anm. 5), S. 194.

45 Vgl. Schmidt (wie Anm. 5), Sp. 1018. Damit war die Einwohnerzahl der Stadt größer als heute mit 2.878 Einwohnern zum 30. Juni 2017 in der Kernstadt Lommatzsch.

46 Vgl. Zahn (wie Anm. 5), S. 113-147.

47 Vgl. Gurlitt (wie Anm. 3), S. 271; Zahn (wie Anm. 5), S. $150 \mathrm{f}$.

48 Vgl. Zahn (wie Anm. 5), S. 155.

49 Vgl. Gernot Härtner: Lommatzsch im Lauf der Jahrhunderte. Festschrift anlässlich 700 Jahre Stadt Lommatzsch 1286-1986. Beiträge zur Geschichte der Stadt, Lommatzsch 1986. Noch vor 80 Jahren sollen sich die Bürger dort frische Milch geholt haben, bis man 1936 die Stallanlagen abriss.

50 Zitat aus dem „Lommatzscher Anzeiger" vom 1. Januar 1858; vgl. auch Vom Brunnen zum Hahn. Der weite Weg des Wassers, Die Geschichte der Wasserversorgung im Versorgungsgebiet der WRG GmbH, Riesa 2013, S. 79-93, hier S. 80 f.

51 Vgl. Vom Brunnen zum Hahn (wie Anm. 50), S. 80.

52 Vgl. Vom Brunnen zum Hahn (wie Anm. 50), S. 8488; Zahn (wie Anm. 5), S. 231-234; zur Verwaltungsstruktur ebenda S. 176: 1873 entschied sich die Stadt Lommatzsch unter die revidierte Städteordnung zu stellen. Zunächst sollte die Städteordnung für kleinere und mittlere Städte genutzt werden. Im Interesse größerer Selbständigkeit entschied sich die Stadt für die Revidierte Städteordnung. Damit gab es einen Stadtrat und ein Stadtverordnetenkollegium.

53 Peter Wunderwald/Reiner Scheffler: Die Bahnlinie RiesaNossen, Eine Zeitreise durch die 125-jährige Geschichte einer der interessantesten Eisenbahnstrecken in Mittelsachsen, Nossen/Oschatz 2004, S. 8.

54 Aus den Firmen Moritz Buschmann und Gotthard \& Kühne entstand der VEB Dämpferbau, den die heutige Firma Lomma Sachsen GmbH als Fundament ansieht.

55 Vgl. Eberhard Büttner (Hrsg.): Firmenchronik Elbtal Tiefkühlkost $\mathrm{GmbH}$, Lommatzsch 1997, S. 4. 
56 Vgl. Keller (wie Anm. 22), S. 297.

57 Vgl. Reiner Groß, Geschichte Sachsens, Leipzig 2001, S. 205-209.

58 Vgl. Keller (wie Anm. 22), S. 303: Die Kleinbauern hatten dagegen unter wachsender Verschuldung zu leiden. Deren rückläufige Zahl lässt Keller auf die Abwanderung aus der Landwirtschaft in die Industrie schließen. Die Gründung von Genossenschaften ist m.E. jedoch gleichermaßen auf wirtschaftliche Schwierigkeiten wie Innovationsdruck zurückzuführen.

59 Vgl. Irmgard Röder: Erntesitten und Erntebräuche in des Lommatzscher Landes, in: Die Lommatzscher Pflege, Heimatbeilage zum Lommatzscher Anzeiger und Tageblatt, 15.08.1935, S. 1-8.

60 Vgl. Gerhard Eidner: Die Eisenbahn in der Lommatzscher Pflege, in: 700 Jahre Stadt Lommatzsch 1286-1986. 1972 wurde sie aus Gründen der Rationalisierung und der Verlagerung des Transports von der Schiene auf die Straße eingestellt. 1998 folgte die Einstellung des Bahnverkehrs auf der Linie Nossen-Lommatzsch-Riesa.

61 Gottfried Ostermay: Die Geschichte unserer Heimatstadt Lommatzsch, in: Schulund Heimatfest Lommatzsch, Lommatzsch 1959, S. 16. Im Einzelnen soll hier nicht näher auf die Ereignisse eingegangen werden. Erschreckende Ergebnisse zeigen die Recherchen der Konfirmanden mit Pfarrer Roland Hartzsch (wie Anm. 2).

\section{Gegenwart (ab 1990)}

Ende des 20. Jahrhunderts stand Lommatzsch vor neuen Herausforderungen. Nach der deutschen Wiedervereinigung erfolgten Schließungen, Privatisierungen von Betriebsteilen und Betriebsübernahmen durch westdeutsche Firmen mit einem deutlichen Abbau von Arbeitsplätzen in den Lommatzscher Betrieben. Auch in der Landwirtschaft gingen durch Reprivatisierungen der Landwirtschaftlichen Produktionsgenossenschaften viele Arbeitsplätze verloren. Zwar fußen die größten Firmen der Stadt (Elbtal Tiefkühlkost GmbH, Scholl Glastechnik $\mathrm{GmbH}$ ) auf traditionellen Lommatzscher Betrieben bzw. wurden wie die Lomma Sachsen $\mathrm{GmbH}$ durch ehemalige Mitarbeiter des VEB Dämpferbaus in die Privatisierung geführt. ${ }^{66} \mathrm{Sie}$ beschäftigen aber aktuell nur etwa ein Viertel der Arbeitskräfte im Vergleich zu DDR-Zeiten. Besonders gravierend war der Verlust von Arbeitsplätzen in den 1990er Jahren in der Landwirtschaft. Gab die LPG „Helmut Just“ in Striegnitz beispielsweise in der DDR rund 600 Personen Arbeit, bewirtschaftet die Nachfolgegenossenschaft, das Agrarunternehmen Lommatzscher Pflege eG, bei rund 3.000 Hektar Feldfläche und rund 1.000 Milchkühen nur noch knapp 60 Mitarbeiter. Es ist damit zugleich das größte Agrarunternehmen der Stadt. Neben diesem bewirtschaften etwa vierzig landwirtschaftliche Betriebe im Haupt- oder Nebengewerbe rund 6.070 Hektar landwirtschaftliche Fläche. Die einzelnen Schläge sind teilweise mehr als 50 Hektar groß. Die Landwirte arbeiten überwiegend konventionell, bauen Getreide, Raps, Zuckerrüben und Gemüse an. Tierproduktion gibt es nur noch wenig. Neben der Milchproduktion des Agrarunternehmens sind die Eierproduktion der Geflügelfarm Lommatzsch in Schwochau mit 130.000 Legehennen und die Sächsische Farmbetriebe $\mathrm{GmbH}$ mit 50.000 Junghennen für die Broilerproduktion zu nennen. Der Landwirtschaftsbetrieb von Judith Faller-Moog in Klappendorf arbeitet als einziges größeres landwirtschaftliches Unternehmen nach den Grundsätzen des biologischen Landbaus.

Die großen Betriebe über 100 Hektar verzeichnen insgesamt eine erfreuliche wirtschaftliche Entwicklung. Gleichwohl bleibt der Arbeitskräftebedarf gering. Die Veredelung landwirtschaftlicher Produkte vor Ort und lokale Wertschöpfungsketten, die mehr Mitarbeiter binden könnten, sind in den letzten Jahrzehnten nicht neu hinzugekommen. Die Produktion der Firma Risse, die Gemüse zu Rohkostsalaten bzw. Weißkraut aus der Region zu Sauerkraut verarbeitet, war bis zur Privatisierung ein Betriebsteil des VEB Elbtal.

Insgesamt prägen viele kleinere Handwerksbetriebe aus dem Bauhaupt- und Baunebengewerk von Dachdeckern und Zimmerern über Elektriker, Malerfirmen bis hin zu Tischlern und Metallbaufirmen die Gewerbelandschaft. Zudem gibt es mehrere Speditionen und ein großes Tiefkühllager in der Stadt. Mit rund 1.500 Arbeitsplätzen könnte statistisch gesehen die Hälfte aller Lommatzscher im erwerbsfähigen Alter mit einem Arbeitsplatz versorgt werden. Faktisch arbeiten rund 700 Lommatzscher am Wohnort. Die Erwerbslosenquote liegt im Landkreis Meißen gegenwärtig bei 7,2 Prozent. Die Gewerbesteuereinnahmen betrugen in den letzten drei Jahren durchschnittlich 1,5 Millionen Euro. Dieser Betrag kann sich für die Größe

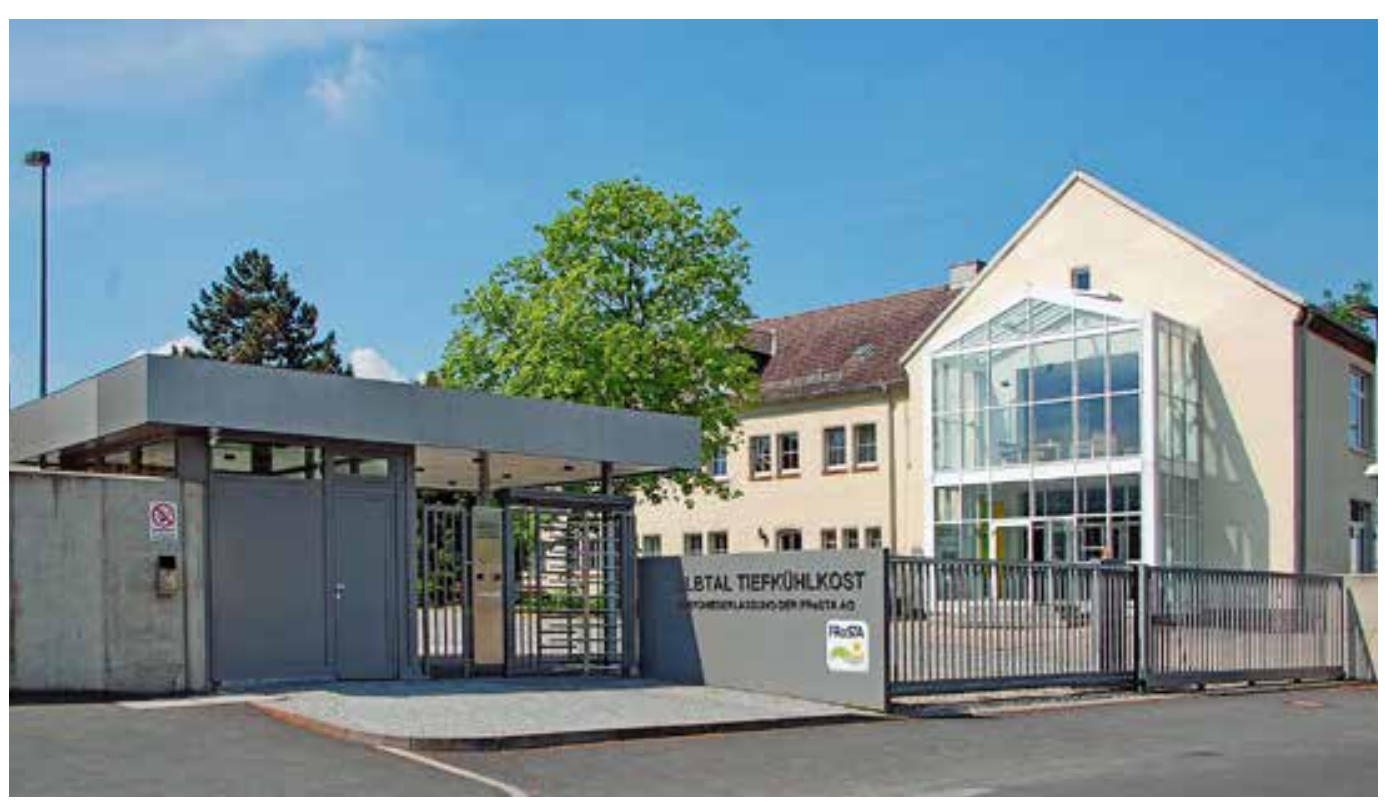




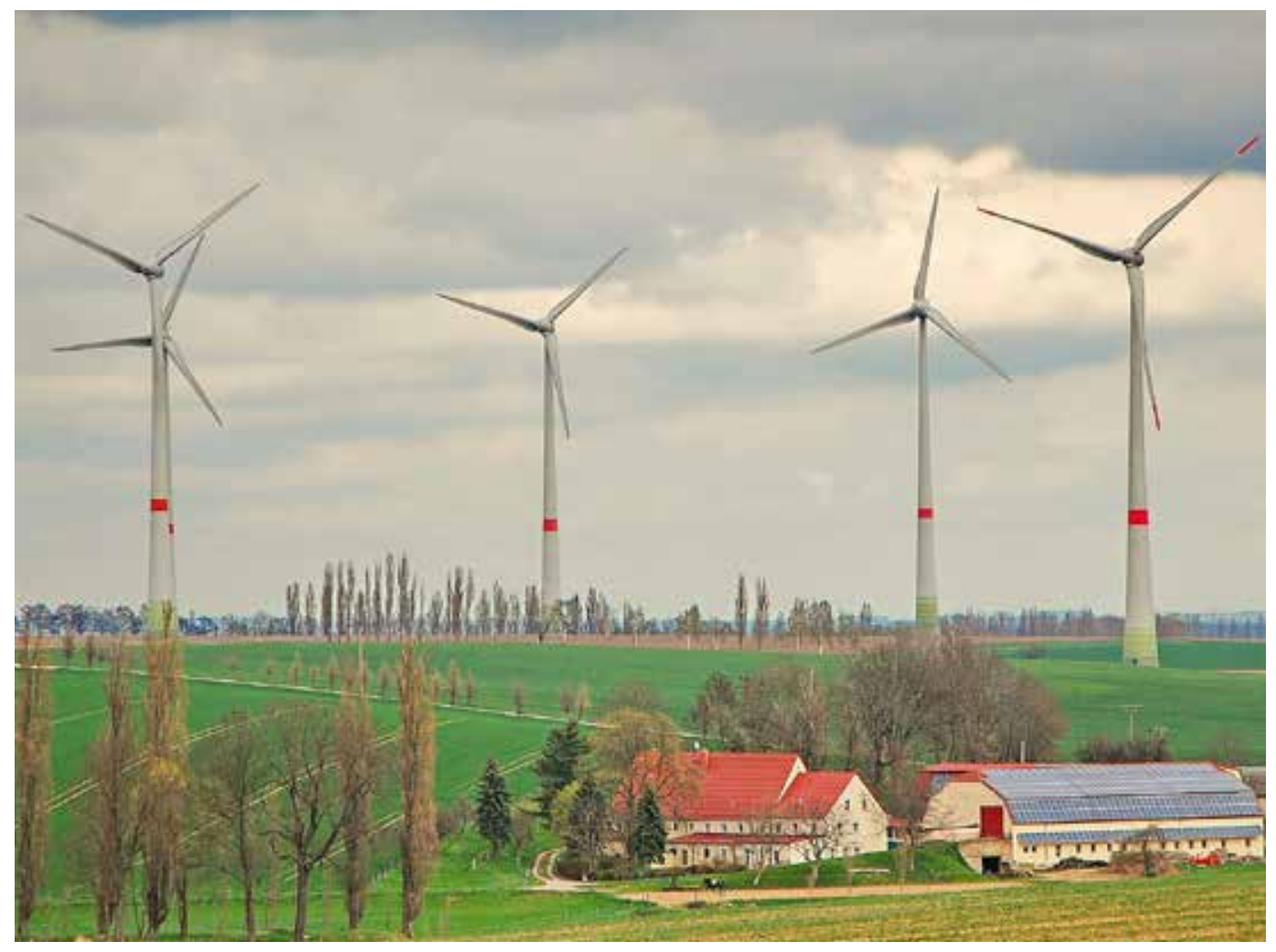

der Stadt durchaus sehen lassen. Demgegenüber sind die Einnahmen aus der Einkommenssteuer mit rund einer Million Euro als vergleichsweise gering einzuschätzen. Diese resultieren aus einer geringen Lohnhöhe in der Gegend, der Überalterung der Einwohner der Stadt mit vielen Rentnern sowie vielen Empfängern sozialer Transferleistungen aufgrund von Arbeitslosigkeit. Insgesamt leidet die Stadt unter einer unzureichenden finanziellen Ausstattung für die Bewältigung ihrer Pflichtaufgaben in einem 66 Quadratkilometer großen Territorium mit 38 Ortsteilen.

Durch den wirtschaftlichen Strukturwandel verlor Lommatzsch seit 1990 bis heute - trotz Eingemeindungen - rund 27 Prozent der Einwohner. Besonders viele junge Menschen (Geburtsjahrgänge zwischen ca. 1970 bis ca. 1985) verließen aufgrund fehlender Ausbildungs- und Arbeitsmöglichkeiten die Stadt. Diese fehlen jetzt, weshalb die jährlich zwischen 30 und 40 Geburten die rund 70 Todesfälle im Jahr nicht auffangen können. Die Abwanderung verläuft aktuell nicht mehr so rasant wie noch vor zehn Jahren. Trotzdem verlassen noch immer Jugendliche, die eine Ausbildung antreten, und betagte Senioren, die in die Nähe ihrer Kinder umziehen, das Stadtgebiet. Insgesamt verliert Lommatzsch jährlich rund 100 Einwohner. Erfreulicherweise steigen die Zuzüge nach Lommatzsch. Deren Zahl ist zwar noch leicht geringer als die Anzahl der Wegzüge, aber verstärkt ziehen jun- ge Familien mit Kindern in die Stadt. Aktuell durchlebt Lommatzsch noch immer einen schmerzhaften Veränderungsprozess. Für ausstehende Betrachter scheinen wirtschaftlicher und demografischer Wandel einen Niedergang der Stadt einzuläuten. Aus historischer Sicht sind die Veränderungen als erklärbarer Prozess im Entwicklungslauf einer jahrtausendealten Kulturlandschaft und einer über 700-jährigen Stadt zu begreifen. Dieser hält neben Risiken auch Chancen für Lommatzsch bereit.

Der Bereich Erneuerbare Energie, mit dem Bau von Biogasanlagen, Solaranlagen und Windrädern, ist beispielsweise in unserer Stadt ein wachsender Wirtschaftszweig. Für die Landwirte sind diese Projekte wichtig, um eigene Energieaufkommen zu sparen oder Abfall- und Reststoffe der Landwirtschaft einer weiteren energetischen Aufbereitung zuzuführen. Zugleich reizt dieser Wirtschaftsbereich einige Bauern durchaus als zweites Standbein. Obgleich die vielen Windräder die Sanftheit der Landschaft optisch durchbrechen, sind sie aktuell wie später wichtige Steuereinnahmequellen für die Stadt.

Der Tourismus hat bisher für die wirtschaftliche Entwicklung der Stadt keine Bedeutung. Lommatzsch ist kein Reiseziel für einen mehrtägigen Aufenthalt und im Vergleich zu den Touristenmagneten Meißen und Dresden überregional nicht bekannt. Trotzdem steigt gerade in der näheren Umgebung das Interesse der
Landwirtschaftliche Flächen und Windräder bei Lommatzsch ๑ Stadtverwaltung Lommatzsch, Foto: Gerhard Schlechte
62 Vgl. Gottfried Bucher: Die Lommatzscher Pflege in Vergangenheit und Gegenwart, in: 700 Jahre Stadt Lommatzsch, Lommatzsch 1986, ohne Seitenzahlen. Nach Katrin Keller gab es in Sachsen im Gegensatz zu Brandenburg oder Mecklenburg den „Juncker“, der über riesige Landgüter verfügte im Vergleich zu landarmen und landlosen Bauern in Sachsen nur ausnahmsweise. Die Großgüter von über 100 Hektar umfassten nur knapp ein Drittel der sächsischen landwirtschaftlichen Fläche und waren noch dazu im Vergleich zu anderen deutschen Gebieten relativ klein. Vgl. Keller (wie Anm. 22), S. 306.

63 Vgl. Keller (wie Anm. 22), S. 308 .

64 Vgl. Bucher (wie Anm. 62). 65 Vgl. Bucher (wie Anm. 62).

661992 kaufte Günter Weidemann die Glastechnik Lommatzsch von der Treuhandanstalt Berlin und gliederte es in seine Firmengruppe Scholl Glastechnik ein. Ebenfalls 1992 wurde der ehemalige VEB Elbtal an die Nordstern Lebensmittel AG Bremerhaven angegliedert. 1990 wurde aus dem ehemaligen Betriebsteil Dämpferbau die Lomma Gerätetechnik $\mathrm{GmbH}$ gegründet. Trotz turbulenter Nachwendegeschichte mit drei Insolvenzen der Nachfolgeunternehmen führt die 2014 neu gegründete Lomma Sachsen $\mathrm{GmbH}$ noch traditionelle Produkte wie die grünen Kraftfahrzeuganhänger für Traktoren im Sortiment. 
Meißner, Riesaer oder Döbelner Bürger an Tagesausflügen in die Lommatzscher Pflege. Zukünftig ist ein regional orientierter Thementourismus zu entwickeln, der unsere Stadt und die Lommatzscher Pflege in Wert setzt. Hierdurch könnten neue Arbeitsplätze im bisher nur sehr schwach ausgeprägten Gastronomie- und Beherbergungsgewerbe sowie als Landschaftsund Gästeführer entstehen.

Und nicht zuletzt wird auch die Landwirtschaft in Zukunft das Erscheinungsbild der Stadt weiter prägen. Gleichzeitig wird sie sich - wie seit Jahrtausenden in der Lommatzscher Pflege verändern. Die Digitalisierung für Precision Farming und Autonomes Fahren landwirtschaftlicher Fahrzeuge wird stärker genutzt werden. Hierfür werden zukünftig noch weniger, dafür gut ausgebildete Arbeitskräfte gebraucht. Doch auch das Bewusstsein für den Natur- und Umweltschutz sowie für den biologischen Anbau von Feldfrüchten, für den Schutz vor Bodenerosion und den Erhalt der natürlichen Flora und Fauna wird sich weiter ausprägen. Damit die Landwirtschaft für die Stadt aber wieder zur wichtigsten wirtschaftlichen Basis werden kann, müssen bundes- oder europapolitisch andere finanzielle Rahmenbedingungen für einen neuen Gleichklang von ländlichem Raum und Landwirtschaft geschaffen werden. Die Gewinne der Landwirtschaft sollten endlich auch wieder vor Ort den Bürgern im ländlichen Raum zu Gute kommen können. Damit würde die nötige Infrastruktur für die Landwirtschaft - von Straßen bis zu Entwässerungsgräben - besser erhalten werden können. Zugleich bekäme Lommatzsch eine wirkliche Chance, sich zu einer kleinen attraktiven Stadt für stressfreies Leben in naturnaher Umgebung abseits der Großstadthektik zu entwickeln.

\section{Zusammenfassung}

Die Entwicklung der Stadt Lommatzsch folgte in der Vergangenheit stets den sich wandelnden Bedürfnissen der sie umgebenden Dörfer und der sich stetig veränderten Landwirtschaft. Handwerk, Handel, Gewerbe und seit dem 19. Jahrhundert die Industrie richteten sich darauf aus, die landwirtschaftlichen Produkte zu verarbeiten, zu verkaufen sowie für die Bedürfnisse der Landwirtschaft zu produzieren. Dies bot zu Zeiten einer manuell geprägten und ar-

Autorin

Dr. Anita Maaß Bürgermeisterin der Stadt

Lommatzsch

Am Markt 1

01623 Lommatzsch
Kleinräumige Wirtschaftskreisläufe zerbrachen im 20. Jahrhundert. Die Orientierung auf die eigene Landwirtschaft reichte für die Stadt nicht mehr aus. In der sozialistischen Planwirtschaft der DDR verursachte diese Entwicklung für die Stadt Lommatzsch keine Probleme. Die Warenströme und der Arbeitskräftebedarf der landwirtschaftlichen Produktion und Verarbeitung, des landwirtschaftlichen Gerätebaus oder auch anderer Produktionsbetriebe wurden zentral gelenkt. Nach 1989/90 jedoch durchlebte die Stadt Lommatzsch in Verbindung mit der sie umgebenden Landwirtschaft einen rasanten und sehr schmerzhaften Wandlungsprozess.

Folglich richteten sich Wachstum und Schrumpfung der Stadt über die Jahrhunderte hinweg stets nach Angebot und Nachfrage von Arbeitsplätzen im Handwerk, Handel, Gewerbe und Landwirtschaft mit dem Bedarf der kleinräumigen Warenkreisläufe. Große strukturelle Veränderungen der Wirtschaft gab es anders als im Erzgebirge (Bergbau) oder im Chemnitzer Raum (Textilindustrie) nicht. Auch die Siedlungsstruktur Lommatzsch als Kleinstadt mit in Feldern eingebetteten kleinen Dörfern bei geringer Bevölkerungsdichte änderte sich kaum. Die Stadt breitete sich nur wenig über ihren ursprünglichen Siedlungskern hinaus aus. Nur die Dörfer Messa und Domselwitz gingen unmittelbar in der Stadtstruktur auf. Damit kann die Landwirtschaft bis heute als wichtigster Faktor der Förderung wie eben auch der Stabilisierung der städtischen Entwicklung gelten. Handwerk und Handel - damit die Nahversorgung - verloren erst in der Gegenwart ihre starke Bindung an die Landwirtschaft.

Als Hemmnisse und Störungen wirkten in der Geschichte vor allem politische, damit äußere Einflüsse wie Kriege, Belagerungen und Epidemien. Doch die Stadt rappelte sich stets wieder auf. Nach schwierigen Zeiten des Niedergangs folgten Phasen des Neuaufbaus und der Fortentwicklung. Ein Verzagen gab es nie. Schließlich gelang Ende des 19./Anfangs des 20. Jahrhunderts durch innovative Fähigkeiten und Risikobereitschaft von Handwerkern, Arbeitern und Bauern vor Ort sogar die Industrialisierung im Gewerbe und der Landwirtschaft. Das kommunalpolitische Streben nach besseren infrastrukturellen Voraussetzungen von der Wasserversorgung bis zur Eisenbahn unterstützte den Fortschritt. Heute wirken viel globalere Faktoren auf die Wirtschaft vor Ort ein. Trotzdem hängt eine gute Entwicklung heute wie damals von „klugen Köpfen“ und „Machern“ in den Firmen, in der Landwirtschaft und in der Stadt ab. Schließlich wird Geschichte wie Zukunft von Menschen gemacht. 